\title{
Partial-wave analysis of two-hadron fragmentation functions
}

\author{
Alessandro Bacchetta用 \\ Division of Physics and Astronomy, Faculty of Science, \\ Free University, NL-1081 HV Amsterdam, the Netherlands \\ Marco Radici门 \\ Dipartimento di Fisica Nucleare e Teorica, Università di Pavia, and \\ Istituto Nazionale di Fisica Nucleare, Sezione di Pavia, I-27100 Pavia, Italy
}

\begin{abstract}
We reconsider the option of extracting the transversity distribution by using interference fragmentation functions into two leading hadrons inside the same current jet. To this end, we perform a new study of two-hadron fragmentation functions. We derive new positivity bounds on them. We expand the hadron pair system in relative partial waves, so that we can naturally incorporate in a unified formalism specific cases already studied in the literature, such as the fragmentation functions arising from the interference between the $s$ - and $p$-wave production of two mesons, as well as the production of a spin-one hadron. In particular, our analysis clearly distinguishes two different ways to access the transversity distribution in two-hadron semi-inclusive leptoproduction.

PACS numbers: 13.87.Fh, 11.80.Et, 13.60.Hb
\end{abstract}

\section{INTRODUCTION}

Two-hadron fragmentation functions have been proposed for the first time in Refs. [1, 2] and then systematically analyzed at leading twist in Ref. [3]. The interest in these functions is mainly justified by the search for a mechanism to single out the chiral-odd transversity distribution ${ }^{1}$ in an alternative and technically simpler way than the Collins effect [5]. In fact, in semi-inclusive deep inelastic scattering (SIDIS) where two unpolarized hadrons are produced in the current fragmentation region, i.e. for the reaction $e p \rightarrow e^{\prime} h_{1} h_{2} X$, it is indeed possible to build a leading-twist singlespin asymmetry (SSA) containing the factorized product of the transversity and a chiral-odd two-hadron fragmentation function [2, 6]. In this process, the asymmetry occurs in the azimuthal angle between the two-hadron plane and the laboratory plane; the total momentum of the hadronic system does not need to have a transverse component, i.e. out of collinearity with respect to the virtual photon axis. Therefore, the intrinsic transverse momentum of the quark can be integrated away and no transverse momentum dependent functions are required, thus introducing simplifications both on the experimental and theoretical side [7] as compared to the Collins effect. Model calculations of such objects are feasible [8] and seem to produce measurable asymmetries in the SIDIS case [6]. Some of the two-hadron fragmentation functions are also naive time-reversal odd (T-odd) and originate from the interference between two production amplitudes with two different phases [3, 5, 9. Therefore, in the literature these functions are usually referred to as interference fragmentation functions (IFF).

In an apparently independent context, semi-inclusive production of spin-1 hadrons (e.g. $\left.\rho, K^{*}, \phi\right)$ has also been studied and proposed as a method to measure the transversity distribution [10, 11, 12, 13]. To measure the polarization of the outgoing vector meson (e.g. $\rho^{0}$ ) it is necessary to measure the 4-momenta of the decay products (e.g. $\pi^{+} \pi^{-}$). Thus, the reaction $e p \rightarrow e^{\prime} \rho^{0} X\left(\rho^{0} \rightarrow \pi^{+} \pi^{-}\right)$is just a part of the more general reaction $e p \rightarrow e^{\prime} \pi^{+} \pi^{-} X$ (namely the part where the total invariant mass of the pion pair is equal to the $\rho$ mass). However, up to now the relation between spin-1 fragmentation functions and two-hadron fragmentation functions has never been thoroughly examined, nor has ever been specified clearly how to access the transversity distribution in the case of spin- 1 fragmentation. The present work is motivated by the need to fill this gap.

Although in our work we focus mainly on SIDIS, two-hadron fragmentation functions can be measured also in $e^{+} e^{-}$annihilation, if hadron pairs belonging to the same jet are identified 14, 15. Some data are already available concerning two hadrons being produced via a spin-1 resonance 116, 17, 18, 19, 20].

The work is organized as follows. In Sec. II, we will review the systematic analysis of semi-inclusive production of

\footnotetext{
*Electronic address: alessandro.bacchetta@physik.uni-regensburg.de; present address: Institut für Theoretische Physik, Universität Regensburg, D-93040 Regensburg, Germany

$\dagger$ Electronic address: radici@pv.infn.it

${ }^{1}$ See Ref. [4] for a review on the topic.
} 
two unpolarized hadrons at leading twist. We will recover the results originally presented in Ref. [3]. We will devote particular attention to the connection with the helicity basis formalism (see, e.g., Refs. [12, 21]) and for the first time we will deduce positivity bounds on IFF.

In Sec. III, the whole problem is reconsidered by expanding in partial waves the two-hadron system in its center-ofmass frame. If we consider only low invariant masses, the expansion can be truncated to include the first two terms only, as hadron pairs are produced mainly in the $s$-wave channel or in the $p$-wave channel (via a spin-1 resonance). We can thus deduce a general unifying formalism that naturally incorporates the specific case of Ref. [2], in the subsector describing the interference between relative $s$ and $p$ waves, as well as the case of spin-1 hadron fragmentation [13], in the subsector of the relative $p$ wave. In particular, we will identify a SSA where the transversity distribution appears in connection with a $s$ - $p$ IFF, and a SSA where the transversity is connected to a pure $p$-wave IFF. These two asymmetries are completely distinct, they could have different physical origins and different magnitudes.

In Sec. [V] we complete our analysis by including the intrinsic partonic transverse momentum and $\vec{k}_{T}$-unintegrated fragmentation functions. Also in this case, in Sec. $\mathrm{V}$ we will present positivity bounds and will carry out the partial wave expansion. The results for the complete cross section for all combinations of beam and target polarizations are listed in the appendices. Finally, some conclusions are drawn in Sec. VI.

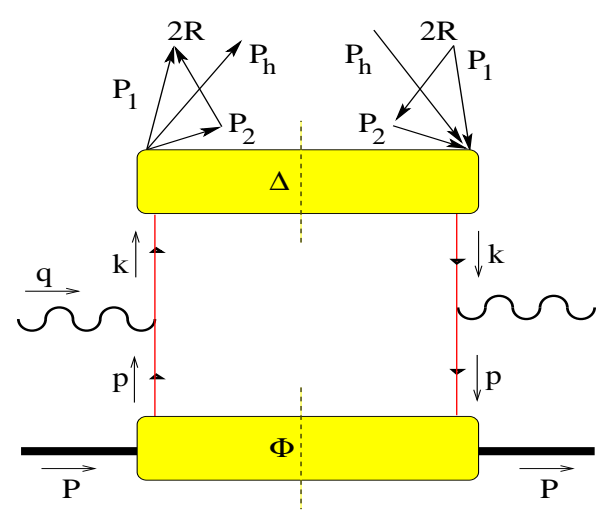

a)

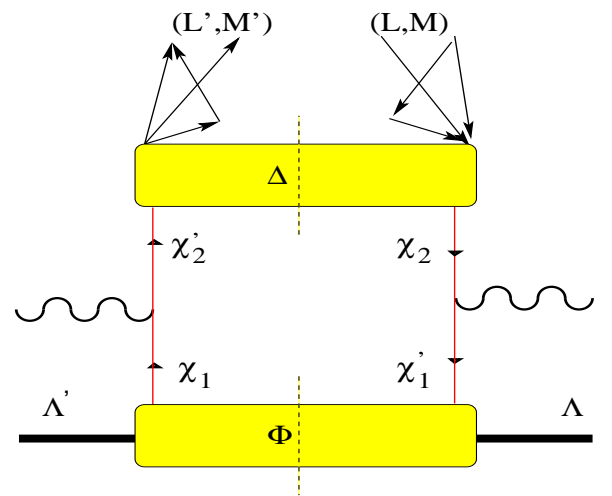

b)

FIG. 1: The usual quark handbag diagram contributing at leading twist to the semi-inclusive DIS into two leading hadrons: a) hadron and parton momenta are shown, in particular the total momentum $P_{h}=P_{1}+P_{2}$ and relative momentum $R=\left(P_{1}-P_{2}\right) / 2$ of the two-hadron system; b) target helicity, parton chirality and two-hadron partial wave indices are shown.

\section{TWO-PARTICLE INCLUSIVE DEEP INELASTIC SCATTERING}

In the following, we will describe the kinematics and the details of the semi-inclusive production of two unpolarized hadrons in the context of the SIDIS process. However, we point out that the involved fragmentation functions can be used also in the case of reactions with a hadronic probe or in $e^{+} e^{-}$annihilation [14, 15].

\section{A. Kinematics and hadronic tensor}

The process is schematically depicted in Fig. 1. An electron with momentum $l$ scatters off a target nucleon with mass $M$, polarization $S$ and momentum $P$, via the exchange of a virtual hard photon with momentum $q=l-l^{\prime}$ $\left(q^{2}=-Q^{2}\right)$. Inside the target, the photon hits a quark with momentum $p$, changing its momentum to $k=p+q$. The quark then fragments into a residual jet and two leading unpolarized hadrons with masses $M_{1}, M_{2}$, and momenta $P_{1}$ and $P_{2}$. We introduce the vectors $P_{h}=P_{1}+P_{2}$ and $R=\left(P_{1}-P_{2}\right) / 2$. We describe a 4 -vector $a$ as $\left[a^{-}, a^{+}, \vec{a}_{T}\right]$, i.e. in terms of its light-cone components $a^{ \pm}=\left(a^{0} \pm a^{3}\right) / \sqrt{2}$ and the bidimensional vector $\vec{a}_{T}$. It is convenient to choose the $\hat{z}$ axis according to the condition $\vec{P}_{T}=\vec{P}_{h T}=0$. In this case, the virtual photon has a nonvanishing transverse momentum $\vec{q}_{T}$. However, it is also customary to align the $\hat{z}$ axis opposite to the direction of the virtual photon, in which case the outgoing hadron has a nonvanishing transverse momentum $\vec{P}_{h \perp}=-z \vec{q}_{T}$. These two directions overlap up to corrections of order $1 / Q$, which we will systematically neglect in the following. The $y$ axis is chosen to point in the direction of the vector product $\left(-\vec{q} \times \overrightarrow{l^{\prime}}\right)$ 22]. 
We define the variables $x=p^{+} / P^{+}$, which represents the light-cone fraction of target momentum carried by the initial quark, and $z=P_{h}^{-} / k^{-}$, the light-cone fraction of fragmenting quark momentum carried by the final hadron pair. Analogously, we define the light-cone fraction $\zeta=2 R^{-} / P_{h}^{-}$, which describes how the total momentum of the hadron pair is split into the two single hadrons. ${ }^{2}$ The relevant momenta can be parametrized as

$$
\begin{aligned}
P^{\mu} & =\left[\frac{M^{2}}{2 P^{+}}, P^{+}, \overrightarrow{0}\right] \\
p^{\mu} & =\left[\frac{p^{2}+\vec{p}_{T}^{2}}{2 x P^{+}}, x P^{+}, \vec{p}_{T}\right] \\
k^{\mu} & =\left[\frac{P_{h}^{-}}{z}, \frac{z\left(k^{2}+\vec{k}_{T}^{2}\right)}{2 P_{h}^{-}}, \vec{k}_{T}\right] \\
P_{h}^{\mu} & =\left[P_{h}^{-}, \frac{M_{h}^{2}}{\left.2 P_{h}^{-}, \overrightarrow{0}\right]}\right. \\
R^{\mu} & =\left[\frac{\zeta}{2} P_{h}^{-}, \frac{\left(M_{1}^{2}-M_{2}^{2}\right)-\frac{\zeta}{2} M_{h}^{2}}{2 P_{h}^{-}}, \vec{R}_{T}\right] .
\end{aligned}
$$

Not all components of the 4 -vectors are independent. In particular, here we observe that

$$
\begin{aligned}
R^{2} & =\frac{M_{1}^{2}+M_{2}^{2}}{2}-\frac{M_{h}^{2}}{4} \\
R_{T}^{2} & =\frac{1}{2}\left[\frac{(1-\zeta)(1+\zeta)}{2} M_{h}^{2}-(1-\zeta) M_{1}^{2}-(1+\zeta) M_{2}^{2}\right] \\
P_{h} \cdot R & =\frac{M_{1}^{2}-M_{2}^{2}}{2} \\
P_{h} \cdot k & =\frac{M_{h}^{2}}{2 z}+z \frac{k^{2}+\left|\vec{k}_{T}\right|^{2}}{2} \\
R \cdot k & =\frac{\left(M_{1}^{2}-M_{2}^{2}\right)-\frac{\zeta}{2} M_{h}^{2}}{2 z}+z \zeta \frac{k^{2}+\left|\vec{k}_{T}\right|^{2}}{4}-\vec{k}_{T} \cdot \vec{R}_{T} .
\end{aligned}
$$

The positivity requirement $R_{T}^{2} \geq 0$ imposes the further constraint

$$
M_{h}^{2} \geq \frac{2}{1+\zeta} M_{1}^{2}+\frac{2}{1-\zeta} M_{2}^{2} .
$$

We shall first consider the case when the cross section is integrated over the transverse momentum of the virtual photon, $\vec{q}_{T}$, postponing the analysis of the complete case in Sec. IV. Until then, no transverse-momentum dependent distribution and fragmentation functions will appear. The seven-fold differential equation for two-particle-inclusive DIS is

$$
\frac{d^{7} \sigma}{d \zeta d M_{h}^{2} d \phi_{R} d z d x d y d \phi_{S}}=\sum_{a} \frac{\alpha^{2} y e_{a}^{2}}{32 z Q^{4}} L_{\mu \nu} 2 M W_{a}^{\mu \nu},
$$

where $L_{\mu \nu}$ is the lepton tensor; $y=\left(E-E^{\prime}\right) / E$ is the fraction of beam energy transferred to the hadronic system and it is related to the lepton scattering angle in the center-of-mass $(\mathrm{cm})$ frame; $\phi_{R}$ and $\phi_{S}$ are the azimuthal angles of $\vec{R}_{T}$ and $\vec{S}_{T}$ with respect to the lepton scattering plane. At tree level, the hadronic tensor for a flavour $a$ is given by

$$
2 M W_{a}^{\mu \nu}=32 z \operatorname{Tr}\left[\Phi_{a}(x, S) \gamma^{\mu} \Delta_{a}\left(z, \zeta, M_{h}^{2}, \phi_{R}\right) \gamma^{\nu}\right]+\left(\begin{array}{c}
q \leftrightarrow-q \\
\mu \leftrightarrow \nu
\end{array}\right)
$$

where

$$
\begin{aligned}
\Phi_{a}(x, S) & =\left.\int d \vec{p}_{T} d p^{-} \Phi_{a}(p ; P, S)\right|_{p^{+}=x P^{+}} \\
\Delta_{a}\left(z, \zeta, M_{h}^{2}, \phi_{R}\right) & =\left.\frac{z}{32} \int d \vec{k}_{T} d k^{+} \Delta_{a}\left(k ; P_{h}, R\right)\right|_{k^{-}=P_{h}^{-} / z}
\end{aligned}
$$

\footnotetext{
${ }^{2}$ Note that $-1 \leq \zeta \leq 1$, and $\zeta=2 \xi-1$, with $\xi$ defined in Ref. [3]
} 
The quark-quark correlator $\Phi$ describes the nonpertubative processes determining the distribution of parton $a$ inside the spin- $1 / 2$ target (represented by the lower shaded blob in Fig. (1) and, similarly, the correlator $\Delta$ symbolizes the fragmentation of quark $a$ producing two tagged leading hadrons in a residual jet (upper shaded blob in Fig. 1).

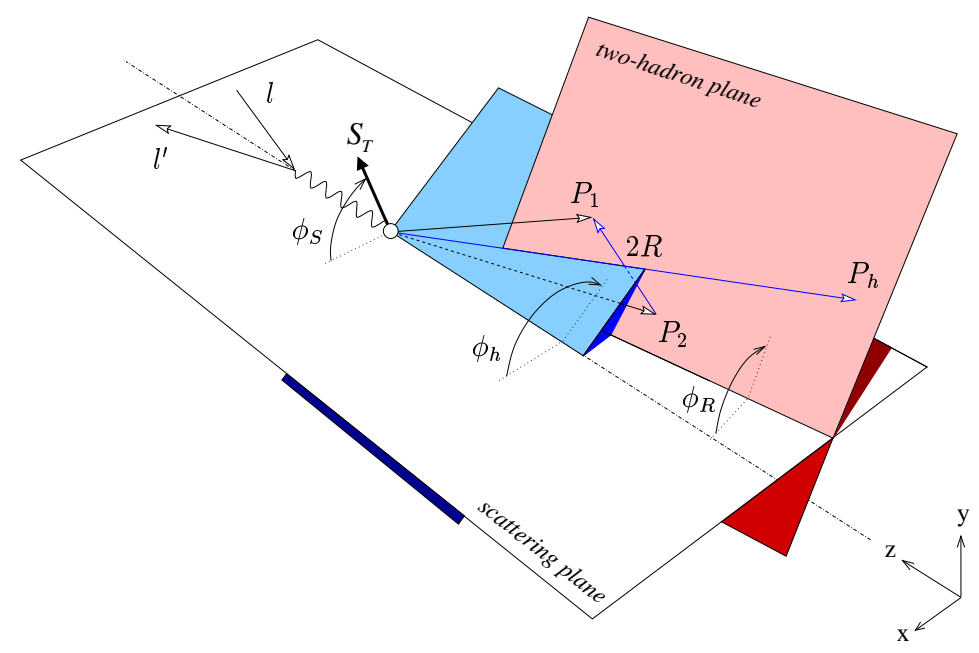

FIG. 2: Kinematics for the SIDIS of the lepton $l$ on a transversely polarized target leading to two hadrons inside the same current jet.

We are going to focus only on the leading twist contributions to the hadronic tensor of Eq. (5). A method to extract these contributions consists in projecting the socalled good light-cone components out of the quark field $\psi$. As it is evident from the kinematics in the infinite momentum frame, the + and the - light-cone components are the dominant ones for the parton entering and exiting the hard vertex, respectively. They can be projected out by means of the operators $\mathcal{P}_{ \pm}=\frac{1}{2} \gamma^{\mp} \gamma^{ \pm}$. Any other component of $\psi$ is automatically of higher twist. Therefore, the hadronic tensor (5) at leading twist looks like

$$
\begin{aligned}
2 M W_{a}^{\mu \nu} & =32 z \operatorname{Tr}\left[\mathcal{P}_{+} \Phi_{a}(x, S) \overline{\mathcal{P}}_{+} \gamma^{\mu} \mathcal{P}_{-} \Delta_{a}\left(z, \zeta, M_{h}^{2}, \phi_{R}\right) \overline{\mathcal{P}}_{-} \gamma^{\nu}\right] \\
& =32 z\left[\mathcal{P}_{+} \Phi_{a}(x, S) \gamma^{+}\right]_{i j}\left[\frac{1}{2} \gamma^{-} \gamma^{\mu} \mathcal{P}_{-}\right]_{j l}\left[\frac{1}{2} \gamma^{+} \gamma^{\nu} \mathcal{P}_{+}\right]_{m i}\left[\mathcal{P}_{-} \Delta_{a}\left(z, \zeta, M_{h}^{2}, \phi_{R}\right) \gamma^{-}\right]_{l m},
\end{aligned}
$$

where $\overline{\mathcal{P}}_{ \pm} \equiv \gamma^{0} \mathcal{P}_{ \pm}^{\dagger} \gamma^{0}$. In the last step the Dirac indices have been explicitly indicated. In the following, we will analyze each contribution to Eq. (8) separately.

\section{B. The quark-quark correlator $\Phi$}

The leading-twist projection of the quark-quark correlator $\Phi$ can be parametrized in terms of the well known distribution functions $[23,24]^{3}$

$$
\begin{aligned}
\mathcal{P}_{+} \Phi_{a}(x, S) \gamma^{+} & =\left(f_{1}^{a}(x)+\lambda g_{1}^{a}(x) \gamma_{5}+2 h_{1}^{a}(x) \gamma_{5} \phi_{T}\right) \mathcal{P}_{+} \\
& =\left(\begin{array}{cccc}
f_{1}^{a}+\lambda g_{1}^{a} & 0 & 0 & \left(S_{x}-i S_{y}\right) h_{1}^{a} \\
0 & 0 & 0 & 0 \\
0 & 0 & 0 & 0 \\
\left(S_{x}+i S_{y}\right) h_{1}^{a} & 0 & 0 & f_{1}^{a}-\lambda g_{1}^{a}
\end{array}\right),
\end{aligned}
$$

where $\lambda=M S^{+} / P^{+}$and $\vec{S}_{T}=\left(S_{x}, S_{y}\right)$ are the light-cone helicity and transverse components of the target spin, respectively $\left(\mathcal{P}_{+} \Phi\right.$ corresponds to the $\vec{p}_{T}$-integrated parametrization of Eq. (2) in Ref. [6]). It is possible to rewrite

\footnotetext{
${ }^{3}$ Other common notations are $f_{1}^{a}(x)=a(x), g_{1}^{a}(x)=\Delta a(x), h_{1}^{a}(x)=\delta a(x), \Delta_{T} a(x)$.
} 
Eq. (9) in a more compact notation, namely in the chiral basis of the good quark fields $\psi_{ \pm R / L}=\mathcal{P}_{ \pm} \mathcal{P}_{R / L} \psi$, with $\mathcal{P}_{R / L}=\left(1 \pm \gamma_{5}\right) / 2$ [24],

$$
\left[\mathcal{P}_{+} \Phi_{a}(x, S) \gamma^{+}\right]_{\chi_{1}^{\prime} \chi_{1}}=\left(\begin{array}{cc}
f_{1}^{a}(x)+\lambda g_{1}^{a}(x) & \left(S_{x}-i S_{y}\right) h_{1}^{a}(x) \\
\left(S_{x}+i S_{y}\right) h_{1}^{a}(x) & f_{1}^{a}(x)-\lambda g_{1}^{a}(x)
\end{array}\right) .
$$

Finally, it is useful to project out also the target helicity density matrix $\rho_{\Lambda \Lambda^{\prime}}$ by

$$
\left[\mathcal{P}_{+} \Phi_{a} \gamma^{+}\right]_{\chi_{1}^{\prime} \chi_{1}}=\rho_{\Lambda \Lambda^{\prime}}\left[\mathcal{P}_{+} \Phi_{a} \gamma^{+}\right]_{\chi_{1}^{\prime} \chi_{1}}^{\Lambda^{\prime} \Lambda}
$$

with

$$
\begin{aligned}
\rho_{\Lambda \Lambda^{\prime}}= & \frac{1}{2}\left(\begin{array}{cc}
1+\lambda & S_{x}-i S_{y} \\
S_{x}+i S_{y} & 1-\lambda
\end{array}\right), \\
{\left[\mathcal{P}_{+} \Phi_{a} \gamma^{+}\right]_{\chi_{1}^{\prime} \chi_{1}}^{\Lambda^{\prime} \Lambda}=} & \left(\begin{array}{cc|cc}
f_{1}^{a}+g_{1}^{a} & 0 & 0 & 0 \\
0 & f_{1}^{a}-g_{1}^{a} & 2 h_{1}^{a} & 0 \\
\hline 0 & 2 h_{1}^{a} & f_{1}^{a}-g_{1}^{a} & 0 \\
0 & 0 & 0 & f_{1}^{a}+g_{1}^{a}
\end{array}\right) .
\end{aligned}
$$

In Eq. (13) the pair of indices $\left(\Lambda, \Lambda^{\prime}\right)$ identifies each component of the $2 \times 2$ submatrices and indicates the spin state of the target; they are attached to each corresponding nucleon leg in the diagram of Fig. 11 b. The pair $\left(\chi_{1}, \chi_{1}^{\prime}\right)$ identifies each submatrix and indicates the parton chirality; they are attached to the emerging quark legs in Fig. 11 b. Equation (13) satisfies general requirements, such as the angular momentum conservation $\left(\chi_{1}+\Lambda=\chi_{1}^{\prime}+\Lambda^{\prime}\right)$, Hermiticity and parity invariance. The chiral transposed matrix is also positive semidefinite, from which the well known Soffer bound [25], among others, is obtained:

$$
\begin{aligned}
f_{1}^{a}(x) & \geq 0 \quad f_{1}^{a}(x) \geq\left|g_{1}^{a}(x)\right| \\
\left|h_{1}^{a}(x)\right| & \leq \frac{1}{2}\left[f_{1}^{a}(x)+g_{1}^{a}(x)\right] .
\end{aligned}
$$

\section{The quark-quark correlator $\Delta$ and positivity bounds}

The most general parametrization of the quark-quark correlator $\Delta\left(k, P_{h}, R\right)$ entering Eq. (7), compatible with Hermiticity and parity invariance, is [3]

$$
\begin{aligned}
\Delta\left(k, P_{h}, R\right)= & M_{h} C_{1} I+C_{2} \not P_{h}+C_{3} \not R+C_{4} \not p \\
& +\frac{C_{5}}{M_{h}} \sigma_{\mu \nu} P_{h}^{\mu} k^{\nu}+\frac{C_{6}}{M_{h}} \sigma_{\mu \nu} R^{\mu} k^{\nu}+\frac{C_{7}}{M_{h}} \sigma_{\mu \nu} P_{h}^{\mu} R^{\nu} \\
& +\frac{C_{8}}{M_{h}^{2}} \gamma_{5} \varepsilon^{\mu \nu \rho \sigma} \gamma_{\mu} P_{h \nu} R_{\rho} k_{\sigma},
\end{aligned}
$$

where the amplitudes $C_{i}\left(k^{2}, k \cdot P_{h}, k \cdot R, R^{2}\right)$ are dimensionless real scalar functions. By using Eqs. (15,7) the leadingtwist projection becomes

$$
\begin{aligned}
& \mathcal{P}_{-} \Delta_{a}\left(z, \zeta, M_{h}^{2}, \phi_{R}\right) \gamma^{-}=\frac{1}{8 \pi}\left(D_{1}^{a}\left(z, \zeta, M_{h}^{2}\right)+i H_{1}^{\varangle a}\left(z, \zeta, M_{h}^{2}\right) \frac{R_{T}}{M_{h}}\right) \mathcal{P}_{-} \\
& =\frac{1}{8 \pi}\left(\begin{array}{cccc}
0 & 0 & 0 & 0 \\
0 & D_{1}^{a} & i e^{i \phi_{R}} \frac{\left|\vec{R}_{T}\right|}{M_{h}} H_{1}^{\varangle a} & 0 \\
0 & -i e^{-i \phi_{R}} \frac{\left|\vec{R}_{T}\right|}{M_{h}} H_{1}^{\varangle a} & D_{1}^{a} & 0 \\
0 & 0 & 0 & 0
\end{array}\right),
\end{aligned}
$$

where

$$
\begin{aligned}
D_{1}\left(z, \zeta, M_{h}^{2}\right) & =\frac{z \pi}{4} \int d^{2} \vec{k}_{T} d k^{2} d\left(2 k \cdot P_{h}\right) \delta\left(\vec{k}_{T}^{2}+k^{2}+\frac{M_{h}^{2}}{z^{2}}-\frac{2 k \cdot P_{h}}{z}\right)\left[C_{2}+\frac{\zeta}{2} C_{3}+\frac{1}{z} C_{4}\right] \\
H_{1}^{\varangle}\left(z, \zeta, M_{h}^{2}\right) & =\frac{z \pi}{4} \int d^{2} \vec{k}_{T} d k^{2} d\left(2 k \cdot P_{h}\right) \delta\left(\vec{k}_{T}^{2}+k^{2}+\frac{M_{h}^{2}}{z^{2}}-\frac{2 k \cdot P_{h}}{z}\right)\left[\frac{1}{z} C_{6}-C_{7}\right] .
\end{aligned}
$$


The prefactors have been chosen to have a better connection with the one-hadron results, i.e. after integrating over $\zeta, M_{h}^{2}$ and $\phi_{R}$. In Eq. (16), $\mathcal{P}_{-} \Delta$ corresponds to the parametrization of Eq.(3) in Ref. [6].

The fragmentation function $H_{1}^{\varangle}$ is chiral odd and represents a possible partner to isolate the transversity distribution inside the cross section at leading twist [6]. Moreover, it is also odd with respect to naive time-reversal transformations (for brevity, T-odd) [3]. Noteworthy, it is the only example of leading-twist T-odd function surviving the integration upon the quark transverse momentum $\vec{k}_{T}$. It would be interesting to investigate it in order to understand what is the relevance of the transverse-momentum dependence in generating T-odd effects [26, 27]. As a consequence, the $\vec{k}_{T}$-integrated $H_{1}^{\varangle}$ could have simpler evolution equations than the ones of the Collins function. Since $H_{1}^{\varangle}$ has the same operator structure as the transversity, it has been suggested that it could have the same evolution equations [28, 29, 30]. However, the situation is complicated by the presence of two hadrons 31$]^{4}$, except for the component of $H_{1}^{\varangle}$ describing the production of a spin-1 resonance (see Sec. IIIB).

Again, in the chiral basis for the good light-cone components Eq. (16) is simplified to

$$
\left[\mathcal{P}_{-} \Delta_{a}\left(z, \zeta, M_{h}^{2}, \phi_{R}\right) \gamma^{-}\right]_{\chi_{2}^{\prime} \chi_{2}}=\frac{1}{8 \pi}\left(\begin{array}{cc}
D_{1}^{a}\left(z, \zeta, M_{h}^{2}\right) & i e^{i \phi_{R}} \frac{\left|\vec{R}_{T}\right|}{M_{h}} H_{1}^{\varangle a}\left(z, \zeta, M_{h}^{2}\right) \\
-i e^{-i \phi_{R}} \frac{\left|\vec{R}_{T}\right|}{M_{h}} H_{1}^{\varangle a}\left(z, \zeta, M_{h}^{2}\right) & D_{1}^{a}\left(z, \zeta, M_{h}^{2}\right)
\end{array}\right),
$$

where $\left(\chi_{2}, \chi_{2}^{\prime}\right)$ are the quark chiralities to be attached to the parton legs entering the $\Delta$ blob in Fig. Ilb.

The matrix in Eq. 19) is positive semi-definite, from which the following bounds can be derived:

$$
D_{1}^{a}\left(z, \zeta, M_{h}^{2}\right) \geq 0 \quad D_{1}^{a}\left(z, \zeta, M_{h}^{2}\right) \geq \frac{\left|\vec{R}_{T}\right|}{M_{h}}\left|H_{1}^{\varangle a}\left(z, \zeta, M_{h}^{2}\right)\right| .
$$

\section{Cross section and transverse spin asymmetry}

Using the previous results, we can now rewrite the leading-twist cross section for unpolarized two-hadron SIDIS in the helicity basis. In fact, after inserting Eqs. (11) and (19) inside Eq. (8), the cross section in Eq. (1) becomes

$$
\frac{d^{7} \sigma}{d \zeta d M_{h}^{2} d \phi_{R} d z d x d y d \phi_{S}}=\sum_{a} \rho_{\Lambda \Lambda^{\prime}}(S)\left[\mathcal{P}_{+} \Phi_{a}(x) \gamma^{+}\right]_{\chi_{1}^{\prime} \chi_{1}}^{\Lambda^{\prime} \Lambda}\left(\frac{d \sigma^{e q_{a}}}{d y}\right)^{\chi_{1} \chi_{1}^{\prime} ; \chi_{2} \chi_{2}^{\prime}}\left[\mathcal{P}_{-} \Delta_{a}\left(z, \zeta, M_{h}^{2}, \phi_{R}\right) \gamma^{-}\right]_{\chi_{2}^{\prime} \chi_{2}}
$$

where

$$
\begin{aligned}
\left(\frac{d \sigma^{e q_{a}}}{d y}\right)^{\chi_{1} \chi_{1}^{\prime} ; \chi_{2} \chi_{2}^{\prime}} & =\frac{e_{a}^{2} \alpha^{2} y}{Q^{4}} L_{\mu \nu}\left(\frac{\gamma^{-} \gamma^{\mu}}{2} \mathcal{P}_{-}\right)^{\chi_{1} \chi_{2}}\left(\frac{\gamma^{+} \gamma^{\nu}}{2} \mathcal{P}_{+}\right)^{\chi_{2}^{\prime} \chi_{1}^{\prime}} \\
& =\frac{2 e_{a}^{2} \alpha^{2}}{Q^{2} y}\left(\begin{array}{cc|ccc}
A(y)+\lambda_{e} \frac{C(y)}{2} & 0 & 0 & -B(y) \\
0 & 0 & 0 & 0 \\
\hline 0 & 0 & 0 & 0 \\
-B(y) & 0 & 0 & A(y)-\lambda_{e} \frac{C(y)}{2}
\end{array}\right)
\end{aligned}
$$

represents the elementary electron-quark scattering. Strictly speaking, this is not a scattering matrix, but a scattering amplitude times the conjugate of a different scattering amplitude [12]. However, for conciseness we follow the notation of Ref. [2]. The polarization of the incident beam is indicated with $\lambda_{e}$ and

$$
A(y)=1-y+\frac{y^{2}}{2}, \quad B(y)=1-y, \quad C(y)=y(2-y) .
$$

In Eq. 22), the indices $\left(\chi_{1}, \chi_{1}^{\prime}\right)$ refer to the chiralities of the entering quarks and identify each submatrix, while $\left(\chi_{2}, \chi_{2}^{\prime}\right)$ refer to the exiting quarks and point to the elements inside each submatrix. By expanding the sum over repeated indices in Eq. (21), we get the expression

$$
\begin{gathered}
\frac{d^{7} \sigma}{d \zeta d M_{h}^{2} d \phi_{R} d z d x d y d \phi_{S}}=\sum_{a} e_{a}^{2} \frac{2 \alpha^{2}}{4 \pi Q^{2} y}\left\{A(y) f_{1}^{a}(x) D_{1}^{a}\left(z, \zeta, M_{h}^{2}\right)+\lambda_{e} \lambda \frac{C(y)}{2} g_{1}^{a}(x) D_{1}^{a}\left(z, \zeta, M_{h}^{2}\right)\right. \\
\left.+B(y) \frac{\left|\vec{S}_{T}\right|\left|\vec{R}_{T}\right|}{M_{h}} \sin \left(\phi_{R}+\phi_{S}\right) h_{1}^{a}(x) H_{1}^{\varangle a}\left(z, \zeta, M_{h}^{2}\right)\right\} .
\end{gathered}
$$

\footnotetext{
${ }^{4}$ We thank D. Boer for pointing out this detail.
} 
For an unpolarized beam $\left(\lambda_{e}=0\right.$, indicated with $\left.O\right)$ and a transversely polarized target $(\lambda=0$, indicated with $T)$, Eq. (24) corresponds to Eq. (10) of Ref. [6] after integrating over all transverse momenta. The following SSA can be built:

$$
\begin{aligned}
A_{O T}^{\sin \left(\phi_{R}+\phi_{S}\right)}\left(y, x, z, M_{h}^{2}\right) & =\frac{\int d \phi_{S} d \phi_{R} d \zeta \sin \left(\phi_{R}+\phi_{S}\right) d^{7} \sigma_{O T}}{\int d \phi_{S} d \phi_{R} d \zeta d^{7} \sigma_{O O}} \\
& =\left|\vec{S}_{T}\right| \frac{B(y)}{A(y)} \frac{\sum_{a} e_{a}^{2} h_{1}^{a}(x) \int d \zeta \frac{\left|\vec{R}_{T}\right|}{2 M_{h}} H_{1}^{\Varangle a}\left(z, \zeta, M_{h}^{2}\right)}{\sum_{a} e_{a}^{2} f_{1}^{a}(x) \int d \zeta D_{1}^{a}\left(z, \zeta, M_{h}^{2}\right)}
\end{aligned}
$$

which allows to isolate the transversity $h_{1}$ at leading twist. Apart from the usual variables $x, y, z$, the only other variable to be measured is the angle $\phi_{R}+\phi_{S}$. Instead of using the scattering plane as a reference to measure azimuthal angles, it is sometimes convenient to use the directions of the beam and of the transverse component of the target spin. The new plane is rotated by the angle $\phi_{S} \equiv-\phi_{l}^{S}$ with respect to the scattering plane; therefore, we have $\phi_{R} \equiv \phi_{R}^{S}-\phi_{l}^{S}$ and $\phi_{R}+\phi_{S} \equiv \phi_{R}^{S}-2 \phi_{l}^{S}[6]$.

The asymmetry described in Eq. (25) is the most general one at leading twist for the case of two-hadron production when an unpolarized lepton beam scatters off a transversely polarized target. No assumptions are made on the behavior of the fragmentation functions. However, as we shall see in the next Section, it is useful and desirable to understand how different partial waves contribute to the above fragmentation functions.

\section{PARTIAL-WAVE EXPANSION FOR THE TWO-HADRON SYSTEM}

If the invariant mass $M_{h}$ of the two hadrons is not very large, the pair can be assumed to be produced mainly in the relative $s$-wave channel, with a typical smooth distribution, or in the $p$-wave channel with a Breit-Wigner profile [32]. Therefore, it is useful to expand Eq. (16) - or equivalently Eq. (19) - in relative partial waves keeping only the first two harmonics. To this purpose, in the following we reformulate the kinematics in the cm frame of the two-hadron system. Then, the leading-twist projection for the quark-quark correlator $\Delta$ is conveniently expanded deducing a more detailed structure than Eq. (19). A set of new bounds is derived and the corresponding expression for the cross section is discussed.

\section{c.m.}

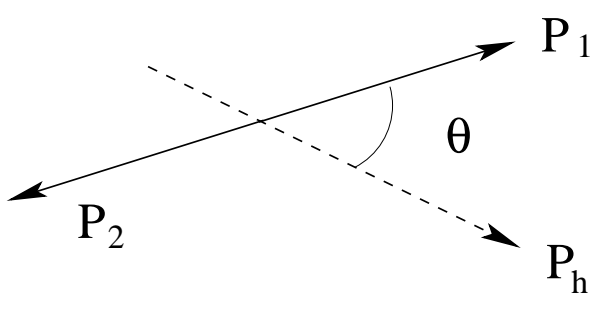

FIG. 3: The hadron pair in the $\mathrm{cm}$ frame; $\theta$ is the $\mathrm{cm}$ polar angle of the pair with respect to the direction of $P_{h}$ in the target rest frame.

In the cm frame the emission of the two hadrons occurs back-to-back. The direction identified by this emission forms an angle $\theta$ with the direction of $P_{h}$ in the target rest frame (see Fig. 3). In this frame, the relevant variables become

$$
\begin{aligned}
P_{h}^{\mu}= & {\left[\frac{M_{h}}{\sqrt{2}}, \frac{M_{h}}{\sqrt{2}}, 0,0\right] } \\
R^{\mu}= & {\left[\frac{\sqrt{M_{1}^{2}+|\vec{R}|^{2}}-\sqrt{M_{2}^{2}+|\vec{R}|^{2}}-2|\vec{R}| \cos \theta}{2 \sqrt{2}}, \frac{\sqrt{M_{1}^{2}+|\vec{R}|^{2}}-\sqrt{M_{2}^{2}+|\vec{R}|^{2}}+2|\vec{R}| \cos \theta}{2 \sqrt{2}}\right.} \\
\left.|\vec{R}| \sin \theta \cos \phi_{R},|\vec{R}| \sin \theta \sin \phi_{R}\right] & \\
\zeta= & \frac{2 R^{-}}{P_{h}^{-}}=\frac{1}{M_{h}}\left(\sqrt{M_{1}^{2}+|\vec{R}|^{2}}-\sqrt{M_{2}^{2}+|\vec{R}|^{2}}-2|\vec{R}| \cos \theta\right)
\end{aligned}
$$


where

$$
|\vec{R}|=\frac{1}{2 M_{h}} \sqrt{M_{h}^{2}-2\left(M_{1}^{2}+M_{2}^{2}\right)+\left(M_{1}^{2}-M_{2}^{2}\right)^{2}} .
$$

The crucial remark is that in this frame $\zeta$ depends linearly on $\cos \theta$, i.e. $\zeta=a+b \cos \theta$, with $a, b$, functions only of the invariant mass. This suggests that any function of $\zeta$ can be conveniently expanded in the basis of Legendre polynomials in $\cos \theta$, as discussed in the following.

\section{A. Partial-wave expansion of the quark-quark correlator $\Delta$ and positivity bounds}

We first express the leading-twist quark-quark correlator (16) in terms of the cm variables. The connection between the two representations is defined as

$$
\Delta\left(z, \cos \theta, M_{h}^{2}, \phi_{R}\right) \equiv \frac{2|\vec{R}|}{M_{h}} \Delta\left(z, \zeta, M_{h}^{2}, \phi_{R}\right)
$$

to take into account the Jacobian of the transformation, $d \zeta=2|\vec{R}| / M_{h} d \cos \theta$. Therefore

$$
\mathcal{P}_{-} \Delta_{a}\left(z, \cos \theta, M_{h}^{2}, \phi_{R}\right) \gamma^{-}=\frac{2|\vec{R}|}{8 \pi M_{h}}\left(D_{1}^{a}\left(z, \zeta(\cos \theta), M_{h}^{2}\right)+i H_{1}^{\varangle a}\left(z, \zeta(\cos \theta), M_{h}^{2}\right) \frac{|\vec{R}|}{M_{h}} \sin \theta \not_{\phi_{R}}\right) \mathcal{P}_{-},
$$

where $\not_{\phi_{R}}=\left[0,0, \cos \phi_{R}, \sin \phi_{R}\right]$.

The fragmentation functions can be expanded in Legendre polynomials as

$$
\begin{aligned}
\frac{2|\vec{R}|}{M_{h}} D_{1}\left(z, \zeta(\cos \theta), M_{h}^{2}\right) & =\sum_{n} D_{1, n}\left(z, M_{h}^{2}\right) P_{n}(\cos \theta) \\
\frac{2|\vec{R}|}{M_{h}} H_{1}^{\varangle}\left(z, \zeta(\cos \theta), M_{h}^{2}\right) & =\sum_{n} H_{1, n}^{\varangle}\left(z, M_{h}^{2}\right) P_{n}(\cos \theta)
\end{aligned}
$$

with

$$
\begin{aligned}
& D_{1, n}\left(z, M_{h}^{2}\right)=\int_{-1}^{1} d \cos \theta P_{n}(\cos \theta) \frac{2|\vec{R}|}{M_{h}} D_{1}\left(z, \zeta(\cos \theta), M_{h}^{2}\right) \\
& H_{1, n}^{\varangle}\left(z, M_{h}^{2}\right)=\int_{-1}^{1} d \cos \theta P_{n}(\cos \theta) \frac{2|\vec{R}|}{M_{h}} H_{1}^{\varangle}\left(z, \zeta(\cos \theta), M_{h}^{2}\right) .
\end{aligned}
$$

We can truncate the expansion to the first three terms only $(n \leq 2)$, which are the minimal set required to describe all the "polarization" states of the system in the $\mathrm{cm}$ frame for relative partial waves with $L=0,1$. In fact, for $n=0$ $\left(P_{0}=1\right)$ the correponding term in the correlator does not depend on $\theta$, it is "unpolarized". For $n=1$, a term linear in $\cos \theta\left(P_{1}=\cos \theta\right)$ describes the interference between an "unpolarized" hadron pair in $s$-wave, for example on the left hand side of Fig. 1 $\mathrm{b}$, and a "longitudinally polarized" pair in $p$-wave on the right hand side. Whenever in the correlator we encounter a term linear in $\sin \theta$, we will interpret it as the interference between a "unpolarized" pair in $s$-wave and a "transversely polarized" pair in $p$-wave. Similarly, a term proportional to $\sin \theta \cos \theta$ indicates the interference between "longitudinally" and "transversely polarized" pairs always in a relative $p$-wave. The last case corresponds to $n=2$, that is interpreted as a "tensor polarization" still related to the intereference between pairs in a relative $p$-wave. With notations that are consistent with previous arguments, the correlator (29) is expanded as

$$
\begin{aligned}
\mathcal{P}_{-} \Delta\left(z, \zeta(\cos \theta), M_{h}^{2}, \phi_{R}\right) \gamma^{-} \sim \frac{1}{8 \pi} & {\left[D_{1,0}\left(z, M_{h}^{2}\right)+D_{1,1}\left(z, M_{h}^{2}\right) \cos \theta+D_{1,2}\left(z, M_{h}^{2}\right) \frac{1}{2}\left(3 \cos ^{2} \theta-1\right)\right.} \\
& \left.+i\left(H_{1,0}^{\varangle}\left(z, M_{h}^{2}\right)+H_{1,1}^{\varangle}\left(z, M_{h}^{2}\right) \cos \theta\right) \sin \theta \frac{|\vec{R}|}{M_{h}} \not h_{\phi_{R}}\right] \mathcal{P}_{-} \\
\equiv \frac{1}{8 \pi} & {\left[D_{1, O O}\left(z, M_{h}^{2}\right)+D_{1, O L}\left(z, M_{h}^{2}\right) \cos \theta+D_{1, L L}\left(z, M_{h}^{2}\right) \frac{1}{4}\left(3 \cos ^{2} \theta-1\right)\right.} \\
& \left.+i\left(H_{1, O T}^{\varangle}\left(z, M_{h}^{2}\right)+H_{1, L T}^{\varangle}\left(z, M_{h}^{2}\right) \cos \theta\right) \sin \theta \frac{|\vec{R}|}{M_{h}} \not h_{\phi_{R}}\right] \mathcal{P}_{-} .
\end{aligned}
$$


Consequently, the same correlator in the chiral basis becomes

$$
\begin{aligned}
& {\left[\mathcal{P}_{-} \Delta\left(z, \zeta, M_{h}^{2}, \phi_{R}\right) \gamma^{-}\right]_{\chi_{2}^{\prime} \chi_{2}} \sim} \\
& \frac{1}{8 \pi}\left(\begin{array}{cc}
D_{1, O O}\left(z, M_{h}^{2}\right)+D_{1, O L}\left(z, M_{h}^{2}\right) \cos \theta & i e^{i \phi_{R}} \frac{|\vec{R}|}{M_{h}} \sin \theta \\
+D_{1, L L}\left(z, M_{h}^{2}\right) \frac{1}{4}\left(3 \cos ^{2} \theta-1\right) & \times\left(H_{1, O T}^{\varangle}\left(z, M_{h}^{2}\right)+H_{1, L T}^{\varangle}\left(z, M_{h}^{2}\right) \cos \theta\right) \\
-i e^{-i \phi_{R}} \frac{|\vec{R}|}{M_{h}} \sin \theta & D_{1, O O}\left(z, M_{h}^{2}\right)+D_{1, O L}\left(z, M_{h}^{2}\right) \cos \theta \\
\times\left(H_{1, O T}^{\varangle}\left(z, M_{h}^{2}\right)+H_{1, L T}^{\varangle}\left(z, M_{h}^{2}\right) \cos \theta\right) & +D_{1, L L}\left(z, M_{h}^{2}\right) \frac{1}{4}\left(3 \cos ^{2} \theta-1\right)
\end{array}\right) .
\end{aligned}
$$

It is useful to project out of Eq. (33) the information about the orbital angular momentum of the system, which is encoded in the angular distribution of the hadron pair. In fact, for $L \leq 1$ the decay matrix for the hadron pair is given by the following bilinear combination of spherical harmonics:

$$
\begin{aligned}
& \mathcal{D}_{M M^{\prime}}^{L L^{\prime}}\left(\theta, \phi_{R}\right)=Y_{L M} Y_{L^{\prime} M^{\prime}}^{*}= \\
& \frac{1}{4 \pi}\left(\begin{array}{c|ccc}
1 & -\sqrt{\frac{3}{2}} \sin \theta e^{i \phi_{R}} & \sqrt{3} \cos \theta & \sqrt{\frac{3}{2}} \sin \theta e^{-i \phi_{R}} \\
\hline-\sqrt{\frac{3}{2}} \sin \theta e^{-i \phi_{R}} & \frac{3}{2} \sin ^{2} \theta & -\frac{3}{\sqrt{2}} \cos \theta \sin \theta e^{-i \phi_{R}} & -\frac{3}{2} \sin ^{2} \theta e^{-2 i \phi_{R}} \\
\sqrt{3} \cos \theta & -\frac{3}{\sqrt{2}} \cos \theta \sin \theta e^{i \phi_{R}} & 3 \cos ^{2} \theta & \frac{3}{\sqrt{2}} \cos \theta \sin \theta e^{-i \phi_{R}} \\
\sqrt{\frac{3}{2}} \sin \theta e^{i \phi_{R}} & -\frac{3}{2} \sin ^{2} \theta e^{2 i \phi_{R}} & \frac{3}{\sqrt{2}} \cos \theta \sin \theta e^{i \phi_{R}} & \frac{3}{2} \sin ^{2} \theta
\end{array}\right),
\end{aligned}
$$

with $L, L^{\prime} \leq 1$ and $\left|M^{(\prime)}\right| \leq L^{(\prime)}$. The upper left block corresponds to $L=L^{\prime}=0$, i.e. to the system being in relative $s$ wave. The lower right block instead corresponds to $L=L^{\prime}=1$, i.e. to the system being in relative $p$ wave, including all the contributions corresponding to different $M, M^{\prime}$ projections and their interferences. The off-diagonal blocks indicate, obviously, the interference between the $s$ and $p$ waves. Using the decay matrix, it is possible to represent the fragmentation in the basis of the quark chirality and of the pair orbital angular momentum. In fact, Eq. (33) can be written as

$$
\left[\mathcal{P}_{-} \Delta\left(z, \zeta, M_{h}^{2}, \phi_{R}\right) \gamma^{-}\right]_{\chi_{2}^{\prime} \chi_{2}}=\left[\mathcal{P}_{-} \Delta\left(z, M_{h}^{2}\right) \gamma^{-}\right]_{M^{\prime} M \chi_{2}^{\prime} \chi_{2}}^{L^{\prime}} \mathcal{D}_{M M^{\prime}}^{L L^{\prime}}\left(\theta, \phi_{R}\right)
$$

where

$$
\left[\mathcal{P}_{-} \Delta\left(z, M_{h}^{2}\right) \gamma^{-}\right]_{M^{\prime} M \chi_{2}^{\prime} \chi_{2}}^{L^{\prime} L}=\frac{1}{8}\left(\begin{array}{cc}
A_{M^{\prime} M}^{L^{\prime} L} & B_{M^{\prime} M}^{L^{\prime} L} \\
\left(B_{M^{\prime} M}^{L^{\prime} L}\right)^{\dagger} & A_{M^{\prime} M}^{L^{\prime} L}
\end{array}\right)
$$

and

$$
\begin{aligned}
A_{M^{\prime} M}^{L^{\prime} L} & =\left(\begin{array}{c|ccc}
D_{1, O O}^{s} & 0 & \frac{2}{\sqrt{3}} D_{1, O L} & 0 \\
\hline 0 & D_{1, O O}^{p}-\frac{1}{3} D_{1, L L} & 0 & 0 \\
\frac{2}{\sqrt{3}} D_{1, O L} & 0 & D_{1, O O}^{p}+\frac{2}{3} D_{1, L L} & 0 \\
0 & 0 & 0 & D_{1, O O}^{p}-\frac{1}{3} D_{1, L L}
\end{array}\right), \\
B_{M^{\prime} M}^{L^{\prime} L} & =\left(\begin{array}{c|ccc}
0 & 0 & i \frac{2 \sqrt{2}}{\sqrt{3}} \frac{|\vec{R}|}{M_{h}} H_{1, O T}^{\varangle} \\
\hline-i \frac{2 \sqrt{2}}{\sqrt{3}} \frac{|\vec{R}|}{M_{h}} H_{1, O T}^{\varangle} & 0 & -i \frac{2 \sqrt{2}}{3} \frac{|\vec{R}|}{M_{h}} H_{1, L T}^{\varangle} & 0 \\
0 & 0 & 0 & i \frac{2 \sqrt{2}}{3} \frac{|\vec{R}|}{M_{h}} H_{1, L T}^{\varangle} \\
0 & 0 & 0 & 0
\end{array}\right) .
\end{aligned}
$$

The fragmentation matrix $\left[\mathcal{P}_{-} \Delta\left(z, M_{h}^{2}\right) \gamma^{-}\right]_{M^{\prime} M \chi_{2}^{\prime} \chi_{2}}^{L^{\prime}}$ fulfills all the fundamental properties, namely Hermiticity, parity invariance [33] and angular momentum conservation $\left(\chi_{2}^{\prime}+M=\chi_{2}+M^{\prime}\right)$. The imaginary entries in its off-diagonal submatrix are T-odd fragmentation functions. It is worth noticing that with the projection (35) we gained a further 
information on the "unpolarized" state of the hadron pair. In fact, we see from the diagonal of Eq. (37) that the spherically symmetric state in the $\mathrm{cm}$ frame receives contributions from both the relative $s$ and $p$ waves, such that when performing the matrix multiplication of Eq. (35) we get

$$
D_{1, O O}\left(z, M_{h}^{2}\right)=\frac{1}{4} D_{1, O O}^{s}\left(z, M_{h}^{2}\right)+\frac{3}{4} D_{1, O O}^{p}\left(z, M_{h}^{2}\right) .
$$

However, in an actual cross section the two contributions are merged together and are kinematically indistinguishable, unless a specific hypothesis on the dependence upon the invariant mass $M_{h}$ is assumed for the two different partial waves, e.g. a resonant contribution for the $p$ wave and a continuum background for the $s$ wave.

Finally, from the matrix (36) being positive semidefinite the following bounds are derived: ${ }^{5}$

$$
\begin{aligned}
D_{1, O O}^{s} & \geq 0, \quad D_{1, O O}^{p} \geq 0, \\
-\frac{3}{2} D_{1, O O}^{p} & \leq D_{1, L L} \leq 3 D_{1, O O}^{p}, \\
D_{1, O L} & \leq \sqrt{\frac{3}{4} D_{1, O O}^{s}\left(D_{1, O O}^{p}+\frac{2}{3} D_{1, L L}\right)} \leq \frac{3}{2} D_{1, O O}, \\
\frac{|\vec{R}|}{M_{h}} H_{1, O T}^{\varangle} & \leq \sqrt{\frac{3}{8} D_{1, O O}^{s}\left(D_{1, O O}^{p}-\frac{1}{3} D_{1, L L}\right)} \leq \frac{3}{2} D_{1, O O}, \\
\frac{|\vec{R}|}{M_{h}} H_{1, L T}^{\varangle} & \leq \frac{3}{2 \sqrt{2}} \sqrt{\left(D_{1, O O}^{p}+\frac{2}{3} D_{1, L L}\right)\left(D_{1, O O}^{p}-\frac{1}{3} D_{1, L L}\right)} \leq \frac{9}{8} D_{1, O O} .
\end{aligned}
$$

\section{B. Cross section and transverse spin asymmetries}

Using Eq. (35) inside Eq. (21), we can take advantage of the full power of the analysis in the helicity formalism. In fact, the cross section can be expanded in the density matrices for the target helicity, for the chirality of the initial and fragmenting quark, and for the relative orbital angular momentum of the leading hadron pair [2]. Inserting the corresponding expressions (12,13,22, 36, 34), we get

$$
\begin{aligned}
& \frac{d^{7} \sigma}{d \zeta d M_{h}^{2} d \phi_{R} d z d x d y d \phi_{S}} \\
& =\sum_{a} \rho_{\Lambda \Lambda^{\prime}}(S)\left[\mathcal{P}_{+} \Phi_{a}(x) \gamma^{+}\right]_{\chi_{1}^{\prime} \chi_{1}}^{\Lambda^{\prime} \Lambda}\left(\frac{d \sigma^{e q a}}{d y}\right)^{\chi_{1} \chi_{1}^{\prime} ; \chi_{2} \chi_{2}^{\prime}}\left[\mathcal{P}_{-} \Delta\left(z, M_{h}^{2}\right) \gamma^{-}\right]_{M^{\prime} M \chi_{2}^{\prime} \chi_{2}}^{L^{\prime} L} \mathcal{D}_{M M^{\prime}}^{L L^{\prime}}\left(\theta, \phi_{R}\right) \\
& =\sum_{a} e_{a}^{2} \frac{\alpha^{2}}{2 \pi Q^{2} y}\left\{\left[A(y) f_{1}^{a}(x)+\lambda_{e} \lambda \frac{C(y)}{2} g_{1}^{a}(x)\right]\left[\frac{D_{1, O O}^{s}+3 D_{1, O O}^{p}}{4}+D_{1, O L} \cos \theta+D_{1, L L} \frac{1}{4}\left(3 \cos ^{2} \theta-1\right)\right]\right. \\
& \left.\quad+B(y) \frac{\left|\vec{S}_{T}\right||\vec{R}|}{M_{h}} \sin \left(\phi_{R}+\phi_{S}\right) h_{1}^{a}(x) \sin \theta\left[H_{1, O T}^{\varangle}+H_{1, L T}^{\varangle} \cos \theta\right]\right\},
\end{aligned}
$$

where all the fragmentation functions depend just on $\left(z, M_{h}^{2}\right)$.

Replacing $\lambda=\lambda_{e}=\left|\vec{S}_{T}\right|=0$ in the previous equation, we get the unpolarized cross section $d^{7} \sigma_{O O}$. However, it is particularly interesting to consider the case for an unpolarized beam and a transversely polarized target, i.e.

$$
\frac{d^{7} \sigma_{O T}}{d \zeta d M_{h}^{2} d \phi_{R} d z d x d y d \phi_{S}}=\sum_{a} e_{a}^{2} \frac{\alpha^{2}}{2 \pi Q^{2} y} B(y) \frac{\left|\vec{S}_{T}\right||\vec{R}|}{M_{h}} \sin \left(\phi_{R}+\phi_{S}\right) h_{1}^{a}(x) \sin \theta\left[H_{1, O T}^{\varangle}+H_{1, L T}^{\varangle} \cos \theta\right],
$$

because we can see that the transversity $h_{1}$ can be matched by two different chiral-odd, T-odd IFF: one $\left(H_{1, O T}^{\varangle}\right)$ pertaining to the interference between $s$ - and $p$-wave states of the hadron pair, the other $\left(H_{1, L T}^{\varangle}\right)$ pertaining to the $p$ wave only. The partial-wave analysis allows us for the first time to comprehend different theoretical analyses in a

\footnotetext{
${ }^{5}$ Note that the bounds involving the pure p-wave functions correspond to those obtained in Ref. [34]
} 
unifying framework. In fact, $H_{1, O T}^{\varangle}$ corresponds to the hypothesis first formulated in Ref. [2], and later reconsidered in Ref. [6], where the necessary spin asymmetry is generated by the interference between two channels describing two leading pions in the relative $s$ and $p$ waves, respectively. As a simple cross-check, taking Eq. (42) and integrating the $\theta$ dependence away yields

$$
\begin{aligned}
\int_{-1}^{1} d \cos \theta \frac{d^{7} \sigma_{O T}}{d \cos \theta d M_{h}^{2} d \phi_{R} d \phi_{S} d z d x d y} & =\int_{-1}^{1} d \cos \theta \frac{d^{7} \sigma_{O T}}{d \zeta d M_{h}^{2} d \phi_{R} d \phi_{S} d z d x d y} \\
& =\sum_{a} e_{a}^{2} \frac{\alpha^{2}}{4 Q^{2} y} B(y) \frac{\left|\vec{S}_{T}\right||\vec{R}|}{M_{h}} \sin \left(\phi_{R}+\phi_{S}\right) h_{1}^{a}(x) H_{1, O T}^{\varangle}\left(z, M_{h}^{2}\right) .
\end{aligned}
$$

This asymmetry corresponds to the one studied in Ref. [2], although in that paper several assumptions were made. Firstly, the IFF was factorized in a part dependent only on the variable $z$, designated as $\delta \hat{q}_{I}(z)$, and in a part containing the $M_{h}$-dependent $\pi-\pi$ phase shifts, $\sqrt{6} \sin \delta_{0} \sin \delta_{1} \sin \left(\delta_{0}-\delta_{1}\right)$. Secondly, the azimuthal angle of the target spin was taken $\phi_{S}=0$, due to neglecting the scattering angle (see Fig. 2). The azimuthal angle of the hadron pair defined in Ref. [2] is $\phi=\pi / 2-\phi_{R}$. It is worth to note that the peculiar behavior in the invariant mass discussed in Ref. 22 relies on the assumption that only the $\pi-\pi$ rescattering can generate the T-odd character of the IFF. It has been already shown, however, that a different model with more general assumptions leads to a unfactorized $\left(z, M_{h}^{2}\right)$ dependence of the fragmentation function and to a completely different behaviour of the SSA [6]. Therefore, it is of great interest to experimentally explore the production of two unpolarized hadrons, e.g. two pions, in the relevant kinematic range, namely with an invariant mass around the $\rho$ resonance.

As for the function $H_{1, L T}^{\varangle}$, it naturally links with the analysis developed in the case of a spin- 1 hadron fragmentation 13, because the two spinless hadrons, e.g., two pions, can be considered as the decay product of a spin-1 resonance, e.g., a $\rho$ particle. The T-odd IFF arise from the interference between two different channels in the relative $p$ wave. To the purpose of isolating an asymmetry containing the function $H_{1, L T}^{\varangle}$, we show that integrating Eq. (42) upon $\theta$ in a different range, namely in the interval $[-\pi / 2, \pi / 2]$, yields

$$
\begin{gathered}
\int_{-\frac{\pi}{2}}^{\frac{\pi}{2}} d \theta \sin \theta \frac{d^{7} \sigma_{O T}}{d \cos \theta d M_{h}^{2} d \phi_{R} d z d x d y d \phi_{S}}=\int_{-\frac{\pi}{2}}^{\frac{\pi}{2}} d \theta \sin \theta \frac{d^{7} \sigma_{O T}}{d \zeta d M_{h}^{2} d \phi_{R} d z d x d y d \phi_{S}} \\
=\sum_{a} e_{a}^{2} \frac{\alpha^{2}}{4 Q^{2} y} B(y) \frac{\left|\vec{S}_{T}\right||\vec{R}|}{M_{h}} \sin \left(\phi_{R}+\phi_{S}\right) h_{1}^{a}(x)\left[H_{1, O T}^{\varangle}\left(z, M_{h}^{2}\right)+\frac{4}{3 \pi} H_{1, L T}^{\varangle}\left(z, M_{h}^{2}\right)\right]
\end{gathered}
$$

where both kinds of IFF appear at leading twist and can contribute to a SSA isolating the transversity $h_{1}$. Although spin-1 fragmentation functions have already been proposed in the past as possible chiral-odd partners for the transversity 110, 11, 12, 13, to our knowledge this is the first time that the asymmetry where they occur is explicitly identified and a clear distinction from the $s-p$ interference is made.

There are not yet quantitative model predictions for $H_{1, L T}^{\varangle}$; on the other hand, since the $p$-wave production of two hadrons becomes significant only when it proceeds via a spin-1 resonance, we can expect that the shape of this function in the invariant mass corresponds to a Breit-Wigner curve peaked at the resonance mass. Moreover, it has the same features as a single-particle fragmentation function, unlike $H_{1, O T}^{\varangle}$ : its evolution equations can be expected to be analogous to that of the transversity [28, 29, 30]; it does not require a rescattering of the hadrons after they are produced and its physical origin could have something in common with the one of the Collins function. However, it should be noticed that in the case of the Collins function an essential role is played by the partonic transverse momentum, which in the case of $H_{1, L T}^{\varangle}$ is replaced by the relative transverse momentum of the hadron pair.

It would be interesting to elaborate on these topics since data for the electromagnetic $\rho$ production and decay are already available in the diffractive regime [35, 36, 37], and they could be available in the DIS regime as well in the near future.

\section{EXPLICIT DEPENDENCE ON THE TRANSVERSE MOMENTA}

For sake of completeness, in this Section we extend the previous results to the case where the transverse momenta are not integrated away. In this case, the cross section is nine-fold and reads

$$
\frac{d^{9} \sigma}{d \zeta d M_{h}^{2} d \phi_{R} d z d \vec{P}_{h \perp} d x d y d \phi_{S}}=\sum_{a} \frac{\alpha^{2} y e_{a}^{2}}{32 z Q^{4}} L_{\mu \nu} 2 M W_{a}^{\mu \nu} .
$$


The hadronic tensor takes the form

$$
2 M W_{a}^{\mu \nu}=32 z \mathcal{I}\left[\operatorname{Tr}\left[\Phi_{a}\left(x, \vec{p}_{T}, S\right) \gamma^{\mu} \Delta_{a}\left(z, \vec{k}_{T}, \zeta, M_{h}^{2}, \phi_{R}\right) \gamma^{\nu}\right]\right]
$$

where we introduced the shorthand notation

$$
\mathcal{I}[f] \equiv \int d \vec{p}_{T} d \vec{k}_{T} \delta\left(\vec{p}_{T}-\vec{P}_{h \perp} / z-\vec{k}_{T}\right)[f],
$$

and where the transverse momentum dependent correlation functions are

$$
\begin{aligned}
\Phi_{a}\left(x, \vec{p}_{T}, S\right) & =\left.\int d p^{-} \Phi_{a}(p ; P, S)\right|_{p^{+}=x P^{+}} \\
\Delta_{a}\left(z, \vec{k}_{T}, \zeta, M_{h}^{2}, \phi_{R}\right) & =\left.\frac{1}{32 z} \int d k^{+} \Delta_{a}\left(k ; P_{h}, R\right)\right|_{k^{-}=P_{h}^{-} / z}
\end{aligned}
$$

The leading-twist projection of $W^{\mu \nu}$ proceeds in an analogous way to Eq. (8); we usually have 22]

$$
\begin{aligned}
\mathcal{P}_{+} \Phi_{a}\left(x, \vec{p}_{T}, S\right) \gamma^{+}= & \left\{f_{1}^{a}\left(x, \vec{p}_{T}^{2}\right)+\frac{\epsilon_{T \rho \sigma} S_{T}^{\rho} p_{T}^{\sigma}}{M} f_{1 T}^{\perp a}\left(x, \vec{p}_{T}^{2}\right)+i h_{1}^{\perp a}\left(x, \vec{p}_{T}^{2}\right) \frac{p_{T}}{M}\right. \\
& +\left[\lambda g_{1 L}^{a}\left(x, \vec{p}_{T}^{2}\right)+\frac{\vec{p}_{T} \cdot \vec{S}_{T}}{M} g_{1 T}^{a}\left(x, \vec{p}_{T}^{2}\right)\right] \gamma_{5} \\
& \left.+\left[\lambda h_{1 L}^{\perp a}\left(x, \vec{p}_{T}^{2}\right)+\frac{\vec{p}_{T} \cdot \vec{S}_{T}}{M} h_{1 T}^{\perp a}\left(x, \vec{p}_{T}^{2}\right)\right] \gamma_{5} \frac{p_{T}}{M}+h_{1 T}^{a}\left(x, \vec{p}_{T}^{2}\right) \gamma_{5} \boldsymbol{S}_{T}\right\},
\end{aligned}
$$

where $\epsilon_{T}^{\mu \nu}=\epsilon^{-+\mu \nu}$. Equation (50) corresponds to Eq.(2) of Ref. [6]. Again, similarly to Eq. (11) and following ones, we project out the density matrix of the target helicity so that Eq. (50) in the basis of quark chirality and target helicity becomes

$$
\begin{gathered}
{\left[\mathcal{P}_{+} \Phi_{a} \gamma^{+}\right]_{\chi_{1}^{\prime} \chi_{1}}^{\Lambda^{\prime}}=} \\
=\left(\begin{array}{cc|cc}
f_{1}^{a}+g_{1 L}^{a} & \frac{\left|\vec{p}_{T}\right|}{M} e^{-i \phi_{p}}\left(g_{1 T}^{a}+i f_{1 T}^{\perp a}\right) & \frac{\left|\vec{p}_{T}\right|}{M} e^{-i \phi_{p}}\left(h_{1 L}^{\perp a}+i h_{1}^{\perp a}\right) & \frac{\left|\vec{p}_{T}\right|^{2}}{M^{2}} e^{-2 i \phi_{p}} h_{1 T}^{\perp a} \\
\frac{\left|\vec{p}_{T}\right|}{M} e^{i \phi_{p}}\left(g_{1 T}^{a}-i f_{1 T}^{\perp a}\right) & f_{1}^{a}-g_{1 L}^{a} & -\frac{\left|h_{T}\right|}{M} e^{-i \phi_{p}}\left(h_{1 L}^{\perp a}-i h_{1}^{\perp a}\right) \\
\hline \frac{\vec{p}_{T} \mid}{M} e^{i \phi_{p}}\left(h_{1 L}^{\perp a}-i h_{1}^{\perp a}\right) & 2 h_{1}^{a} & f_{1}^{a}-g_{1 L}^{a} & -\frac{\left|\vec{p}_{T}\right|}{M} e^{-i \phi_{p}}\left(g_{1 T}^{a}-i f_{1 T}^{\perp a}\right) \\
\frac{\left|\vec{p}_{T}\right|^{2}}{M^{2}} e^{2 i \phi_{p}} h_{1 T}^{\perp a} & -\frac{\left|\vec{p}_{T}\right|}{M} e^{i \phi_{p}}\left(h_{1 L}^{\perp a}+i h_{1}^{\perp a}\right) & -\frac{\left|\vec{p}_{T}\right|}{M} e^{i \phi_{p}}\left(g_{1 T}^{a}+i f_{1 T}^{\perp a}\right) & f_{1}^{a}+g_{1 L}^{a}
\end{array}\right)
\end{gathered}
$$

where $\phi_{p}$ is the azimuthal angle of $\vec{p}_{T}$. The matrix is Hermitean, respects parity invariance and conservation of total angular momentum. Introducing the dependence upon the quark transverse momentum $\vec{p}_{T}$ modifies the conditions for angular momentum and parity conservation, which now read, respectively,

$$
\begin{aligned}
\Lambda_{1}^{\prime}+\chi_{1}^{\prime}+l_{p_{T}} & =\Lambda_{1}+\chi_{1} \\
{\left[\mathcal{P}_{+} \Phi \gamma^{+}\right]_{\chi_{1}^{\prime} \chi_{1}}^{\Lambda^{\prime} \Lambda} } & =\left.(-1)^{l_{p_{T}}}\left[\mathcal{P}_{+} \Phi \gamma^{+}\right]_{-\chi_{1}-\chi_{1}^{\prime}}^{-\Lambda^{\prime}-\Lambda}\right|_{l_{p_{T}} \rightarrow-l_{p_{T}}}
\end{aligned}
$$

where $l_{p_{T}}$ denotes the units of angular momentum introduced by $\vec{p}_{T}$. The chiral transposed matrix is still positive definite, so that the bounds on the various distribution functions can be obtained [24].

The leading-twist projection of the fragmenting quark correlator is

$$
\begin{aligned}
\mathcal{P}_{-} \Delta_{a}\left(z, \vec{k}_{T}, \zeta,\right. & \left.M_{h}^{2}, \phi_{R}\right) \gamma^{-}=\frac{1}{8 \pi}\left(D_{1}^{a}\left(z, \zeta, M_{h}^{2}, \vec{k}_{T}^{2}, \vec{k}_{T} \cdot \vec{R}_{T}\right)+i \bar{H}_{1}^{\Varangle a}\left(z, \zeta, M_{h}^{2}, \vec{k}_{T}^{2}, \vec{k}_{T} \cdot \vec{R}_{T}\right) \frac{R_{T}}{M_{h}}\right. \\
& \left.+i H_{1}^{\perp a}\left(z, \zeta, M_{h}^{2}, \vec{k}_{T}^{2}, \vec{k}_{T} \cdot \vec{R}_{T}\right) \frac{\not k_{T}}{M_{h}}+G_{1}^{\perp a}\left(z, \zeta, M_{h}^{2}, \vec{k}_{T}^{2}, \vec{k}_{T} \cdot \vec{R}_{T}\right) \frac{\epsilon_{T}^{\mu \nu} R_{T \mu} k_{T \nu}}{M_{h}^{2}} \gamma_{5}\right) \mathcal{P}_{-}
\end{aligned}
$$

where the actual dependence of the fragmentation functions is the most general one possible [3]. In Eq. (53]) $\mathcal{P}_{-} \Delta_{a}$ corresponds to Eq.(3) of Ref. [6]. New functions appear: $G_{1}^{\perp}$ is chiral even but T-odd, $H_{1}^{\perp}$ is chiral odd and T-odd and represents the analogue of the Collins effect for a two-hadron emission 3. Upon integration over $d \vec{k}_{T}$, $G_{1}^{\perp}$ vanishes 
and the surviving parts of $\bar{H}_{1}^{\varangle}$ and $H_{1}^{\perp}$ merge into the function $H_{1}^{\varangle}$ of Eq. (16) keeping the same $\not R_{T} / M_{h}$ structure. In the chiral basis of the fragmenting quark, Eq. (53) becomes

$$
\left[\mathcal{P}_{-} \Delta_{a}\left(z, \vec{k}_{T}, \zeta, M_{h}^{2}, \phi_{R}\right) \gamma^{-}\right]_{\chi_{2}^{\prime} \chi_{2}}=\frac{1}{8 \pi}\left(\begin{array}{cc}
D_{1}^{a}+\frac{\epsilon_{T}^{\mu \nu} R_{T \mu} k_{T \nu}}{M_{h}^{2}} G_{1}^{\perp a} & i\left(e^{i \phi_{R}} \frac{\left|\vec{R}_{T}\right|}{M_{h}} \bar{H}_{1}^{\varangle a}+e^{i \phi_{k}} \frac{\left|\vec{k}_{T}\right|}{M_{h}} H_{1}^{\perp a}\right) \\
-i\left(e^{-i \phi_{R}} \frac{\left|\vec{R}_{T}\right|}{M_{h}} \bar{H}_{1}^{\varangle a}+e^{-i \phi_{k}} \frac{\left|\vec{k}_{T}\right|}{M_{h}} H_{1}^{\perp a}\right) & D_{1}^{a}-\frac{\epsilon_{T}^{\mu \nu} R_{T \mu} k_{T \nu}}{M_{h}^{2}} G_{1}^{\perp a}
\end{array}\right) .
$$

The following bounds are derived:

$$
\begin{gathered}
\frac{\left|\epsilon_{T}^{\mu \nu} R_{T \mu} k_{T \nu}\right|}{M_{h}^{2}}\left|G_{1}^{\perp a}\right| \leq D_{1}^{a} \\
\frac{\left|\vec{R}_{T}\right|^{2}}{M_{h}^{2}}\left(\bar{H}_{1}^{\varangle a}\right)^{2}+\frac{\left|\vec{k}_{T}\right|^{2}}{M_{h}^{2}}\left(H_{1}^{\perp a}\right)^{2}+\frac{2 \vec{k}_{T} \cdot \vec{R}_{T}}{M_{h}^{2}} \bar{H}_{1}^{\varangle a} H_{1}^{\perp a} \leq\left(D_{1}^{a}\right)^{2}-\frac{\left|\epsilon_{T}^{\mu \nu} R_{T \mu} k_{T \nu}\right|^{2}}{M_{h}^{4}}\left|G_{1}^{\perp a}\right|^{2} .
\end{gathered}
$$

Expanding the cross section of Eq. (45) along the same lines leading to Eq. (21), we have

$$
\begin{aligned}
\frac{d^{9} \sigma}{d \zeta d M_{h}^{2} d \phi_{R} d \vec{P}_{h \perp} d z d x d y d \phi_{S}}=\sum_{a} & \rho_{\Lambda \Lambda^{\prime}}(S) \mathcal{I}\left[\left[\mathcal{P}_{+} \Phi_{a}\left(x, \vec{p}_{T}\right) \gamma^{+}\right]_{\chi_{1}^{\prime} \chi_{1}}^{\Lambda^{\prime} \Lambda}\left(\frac{d \sigma^{e q_{a}}}{d y}\right)^{\chi_{1} \chi_{1}^{\prime} ; \chi_{2} \chi_{2}^{\prime}}\right. \\
& \left.\times\left[\mathcal{P}_{-} \Delta_{a}\left(z, \vec{k}_{T}, \zeta, M_{h}^{2}, \phi_{R}\right) \gamma^{-}\right]_{\chi_{2}^{\prime} \chi_{2}}\right]
\end{aligned}
$$

where $\left[\mathcal{P}_{+} \Phi_{a} \gamma^{+}\right]$and $\left[\mathcal{P}_{-} \Delta_{a} \gamma^{-}\right]$are given by Eqs. (51) and (54), respectively. The complete formula for the cross section is given in App. A.

\section{PARTIAL-WAVE EXPANSION WITH TRANSVERSE MOMENTA}

It is again useful to expand all the fragmentation functions of Eq. (53) in the relative partial waves of the hadron pair. The dependence on $\vec{k}_{T} \cdot \vec{R}_{T}$ makes the expansion more involved:

$$
\begin{gathered}
D_{1}=D_{1, O O}+D_{1, O L} \cos \theta+D_{1, L L} \frac{1}{4}\left(3 \cos ^{2} \theta-1\right)+\cos \left(\phi_{k}-\phi_{R}\right) \sin \theta\left(D_{1, O T}+D_{1, L T} \cos \theta\right) \\
\quad+\cos \left(2 \phi_{k}-2 \phi_{R}\right) \sin ^{2} \theta D_{1, T T} \\
G_{1}^{\perp}=G_{1, O T}^{\perp}+G_{1, L T}^{\perp} \cos \theta+\cos \left(\phi_{k}-\phi_{R}\right) \sin \theta G_{1, T T}^{\perp} \\
\bar{H}_{1}^{\varangle}=\bar{H}_{1, O T}^{\varangle}+\bar{H}_{1, L T}^{\varangle} \cos \theta+2 \cos \left(2 \phi_{k}-2 \phi_{R}\right) \sin \theta \bar{H}_{1, T T}^{\varangle} \\
H_{1}^{\perp}=H_{1, O O}^{\perp}+H_{1, O L}^{\perp} \cos \theta+H_{1, L L}^{\perp} \frac{1}{4}\left(3 \cos ^{2} \theta-1\right)+2 \cos \left(\phi_{k}-\phi_{R}\right) \sin \theta\left(H_{1, O T}^{\perp}+H_{1, L T}^{\perp} \cos \theta\right) \\
\quad+2 \cos \left(2 \phi_{k}-2 \phi_{R}\right) \sin ^{2} \theta H_{1, T T}^{\perp}-\sin ^{2} \theta \frac{|\vec{R}|}{\left|\vec{k}_{T}\right|} H_{1, T T}^{\varangle},
\end{gathered}
$$

where all the functions depend on $\left(z, \vec{k}_{T}^{2}, M_{h}^{2}\right)$. Then, similarly to Eq. (35), Eq. (54) can be further expanded in the basis of the pair orbital angular momentum as

$$
\left[\mathcal{P}_{-} \Delta\left(z, \vec{k}_{T}, \zeta, M_{h}^{2}, \phi_{R}\right) \gamma^{-}\right]_{\chi_{2}^{\prime} \chi_{2}}=\left[\mathcal{P}_{-} \Delta\left(z, \vec{k}_{T}^{2}, M_{h}^{2}\right) \gamma^{-}\right]_{M^{\prime} M \chi_{2}^{\prime} \chi_{2}}^{L^{\prime}} \mathcal{D}_{M M^{\prime}}^{L L^{\prime}}\left(\theta, \phi_{k}, \phi_{R}\right) .
$$

The full expression of $\left[\mathcal{P}_{-} \Delta\left(z, \vec{k}_{T}^{2}, M_{h}^{2}\right) \gamma^{-}\right]$is shown in App. B. The fully expanded differential cross section in the helicity basis of target, initial and final quark, as well as in the basis of orbital angular momentum of the hadron pair is then

$$
\begin{aligned}
\frac{d^{9} \sigma}{d \zeta d M_{h}^{2} d \phi_{R} d \vec{P}_{h \perp} d z d x d y d \phi_{S}}=\sum_{a} & \rho_{\Lambda \Lambda^{\prime}}(S) \mathcal{I}\left[\left[\mathcal{P}_{+} \Phi_{a}\left(x, \vec{p}_{T}\right) \gamma^{+}\right]_{\chi_{1}^{\prime} \chi_{1}}^{\Lambda^{\prime} \Lambda}\left(\frac{d \sigma^{e q_{a}}}{d y}\right)^{\chi_{1} \chi_{1}^{\prime} ; \chi_{2} \chi_{2}^{\prime}}\right. \\
& \left.\times\left[\mathcal{P}_{-} \Delta_{a}\left(z, \vec{k}_{T}, \zeta, M_{h}^{2}, \phi_{R}\right) \gamma^{-}\right]_{M^{\prime} M \chi_{2}^{\prime} \chi_{2}}^{L^{\prime}}\right] \mathcal{D}_{M M^{\prime}}^{L L^{\prime}}\left(\theta, \phi_{R}\right) .
\end{aligned}
$$

Its explicit expression is presented in App. Q. The pure $p$-wave sector corresponds to the cross section for the production of a polarized spin-1 hadron and has already been fully studied in Refs. [13, 38]. For sake of completeness, we show it in App. C together with the formulae for the pure $s$ and $s-p$ interference sectors. 


\section{CONCLUSIONS}

In this paper, we have reconsidered the option of extracting the transversity distribution $h_{1}$ at leading twist by using the analyzing power of the interference fragmentation functions (IFF) into two leading unpolarized hadrons inside the same current jet. As already shown in Ref. [6], in the process $e p^{\uparrow} \rightarrow e^{\prime} h_{1} h_{2} X$ the transversity distribution enters a single-spin asymmetry in the azimuthal angle $\phi_{R}$ of the hadron pair plane. The effect survives after the integration upon the transverse component of $P_{h}=P_{1}+P_{2}$. Therefore, no transverse-momentum dependent function is required and the advantage with respect to the Collins effect is evident. A similar situation was known to occurr in the case of fragmentation into spin-1 hadrons [10, 11, 12, 13], but it was never fully examined to the extent of defining a specific asymmetry.

Here, we have reanalyzed the whole problem in the helicity formalism by further expanding the IFF in the basis of the relative orbital angular momentum in the $\mathrm{cm}$ frame of the hadron pair. New positivity bounds have been derived. If the invariant mass of the pair is not large, the expansion can be limited to the first two modes, namely the relative $s$ and $p$ waves.

Off-diagonal elements in the chirality and in the orbital angular momentum $L$ represent the IFF of Ref. [2] and [6], where the interference arises from the hadron pair being in a state with either $s$ or $p$ relative wave. Elements in the $L=L^{\prime}=1$ sector correspond to the analysis of spin-1 hadron fragmentation [13]. Therefore, the present formalism represents a unifying framework for the problem of fragmentation into two unpolarized hadrons and can be used to correctly and exhaustively discuss the extraction of transversity from two-hadron leptoproduction.

In fact, after calculating the complete leading-twist cross section, we have identified a single spin asymmetry containing two distinct chiral-odd partners of the transversity. By integrating the asymmetry over different ranges of the cm polar angle of the hadron pair, the transversity $h_{1}$ can be extracted through the chiral-odd, T-odd fragmentation $H_{1, O T}^{\varangle}$ (corresponding to the $s$ - $p$ interference of Ref. [2]) or through the chiral-odd, T-odd fragmentation $H_{1, L T}^{\varangle}$ (corresponding to the $p$ - $p$ interference). This second option has been often neglected in the literature, despite the fact that the two functions have, in principle, a different dependence on the invariant mass and a different physical origin.

In conclusion, we believe that the fragmentation into two leading unpolarized hadrons can be a promising tool to measure the transversity distribution, as well as to achieve further comprehension of the hadronization mechanism.

\section{Acknowledgments}

Several discussions with Daniel Boer are gratefully acknowledged. This work has been supported by the TMR network HPRN-CT-2000-00130.

\section{APPENDIX A}

In this appendix, we write explicitly the cross section for two-hadron leptoproduction at leading order in $1 / Q$ and with the inclusion of partonic transverse momenta. Moreover, we include also T-odd distribution functions, since recently there have been some indications that they are not forbidden by time-invariance [26, 27, 39]. To simplify the notation, we introduce the projection $\vec{a}_{T} \wedge \vec{b}_{T}=a_{i} \epsilon_{T}^{i j} b_{j}$. Inserting in Eq. (45) the formulae for the target helicity density matrix, Eq. (12), for the distribution correlation matrix, Eq. (51), for the elementary scattering matrix, Eq. (22), and the two-hadron fragmentation matrix, Eq. (54), we obtain the following result:

$$
\begin{aligned}
d^{9} \sigma_{O O}= & \sum_{a} \frac{\alpha^{2} e_{a}^{2}}{2 \pi Q^{2} y}\left\{A(y) \mathcal{I}\left[f_{1} D_{1}\right]-B(y) \frac{\left|\vec{R}_{T}\right|}{M_{h}} \cos \left(\phi_{h}+\phi_{R}\right) \mathcal{I}\left[\frac{\vec{p}_{T} \cdot \hat{P}_{h \perp}}{M} h_{1}^{\perp} \bar{H}_{1}^{\varangle}\right]\right. \\
& +B(y) \frac{\left|\vec{R}_{T}\right|}{M_{h}} \sin \left(\phi_{h}+\phi_{R}\right) \mathcal{I}\left[\frac{\hat{P}_{h \perp} \wedge \vec{p}_{T}}{M} h_{1}^{\perp} \bar{H}_{1}^{\Varangle}\right] \\
& -B(y) \cos \left(2 \phi_{h}\right) \mathcal{I}\left[\frac{2\left(\vec{p}_{T} \cdot \hat{P}_{h \perp}\right)\left(\vec{k}_{T} \cdot \hat{P}_{h \perp}\right)-\vec{p}_{T} \cdot \vec{k}_{T}}{M M_{h}} h_{1}^{\perp} H_{1}^{\perp}\right] \\
& \left.+B(y) \sin \left(2 \phi_{h}\right) \mathcal{I}\left[\frac{\left(\vec{p}_{T} \cdot \hat{P}_{h \perp}\right)\left(\hat{P}_{h \perp} \wedge \vec{k}_{T}\right)+\left(\vec{k}_{T} \cdot \hat{P}_{h \perp}\right)\left(\hat{P}_{h \perp} \wedge \vec{p}_{T}\right)}{M M_{h}} h_{1}^{\perp} H_{1}^{\perp}\right]\right\} \\
d^{9} \sigma_{L O}= & -\sum_{a} \frac{\alpha^{2} e_{a}^{2}}{2 \pi Q^{2} y}\left|\lambda_{e}\right| C(y) \frac{\left|\vec{R}_{T}\right|}{M_{h}}\left\{\sin \left(\phi_{h}-\phi_{R}\right) \mathcal{I}\left[\frac{\vec{k}_{T} \cdot \hat{P}_{h \perp}}{M_{h}} f_{1} G_{1}^{\perp}\right]\right.
\end{aligned}
$$




$$
\begin{aligned}
& \left.+\cos \left(\phi_{h}-\phi_{R}\right) \mathcal{I}\left[\frac{\hat{P}_{h \perp} \wedge \vec{k}_{T}}{M_{h}} f_{1} G_{1}^{\perp}\right]\right\} \\
d^{9} \sigma_{O L}= & \sum_{a} \frac{\alpha^{2} e_{a}^{2}}{2 \pi Q^{2} y}\left|S_{L}\right|\left\{-A(y) \frac{\left|\vec{R}_{T}\right|}{M_{h}} \sin \left(\phi_{h}-\phi_{R}\right) \mathcal{I}\left[\frac{\vec{k}_{T} \cdot \hat{P}_{h \perp}}{M_{h}} g_{1 L} G_{1}^{\perp}\right]\right. \\
& -A(y) \frac{\left|\vec{R}_{T}\right|}{M_{h}} \cos \left(\phi_{h}-\phi_{R}\right) \mathcal{I}\left[\frac{\hat{P}_{h \perp} \wedge \vec{k}_{T}}{M_{h}} g_{1 L} G_{1}^{\perp}\right] \\
& +B(y) \frac{\left|\vec{R}_{T}\right|}{M_{h}} \sin \left(\phi_{h}+\phi_{R}\right) \mathcal{I}\left[\frac{\vec{p}_{T} \cdot \hat{P}_{h \perp}}{M} h_{1 L}^{\perp} \bar{H}_{1}^{\varangle}\right] \\
& +B(y) \frac{\left|\vec{R}_{T}\right|}{M_{h}} \cos \left(\phi_{h}+\phi_{R}\right) \mathcal{I}\left[\frac{\hat{P}_{h \perp} \wedge \vec{p}_{T}}{M} h_{1 L}^{\perp} \bar{H}_{1}^{\varangle}\right] \\
& +B(y) \sin \left(2 \phi_{h}\right) \mathcal{I}\left[\frac{2\left(\vec{p}_{T} \cdot \hat{P}_{h \perp}\right)\left(\vec{k}_{T} \cdot \hat{P}_{h \perp}\right)-\vec{p}_{T} \cdot \vec{k}_{T}}{M M_{h}} h_{1 L}^{\perp} H_{1}^{\perp}\right] \\
& \left.+B(y) \cos \left(2 \phi_{h}\right) \mathcal{I}\left[\frac{\left(\vec{p}_{T} \cdot \hat{P}_{h \perp}\right)\left(\hat{P}_{h \perp} \wedge \vec{k}_{T}\right)+\left(\vec{k}_{T} \cdot \hat{P}_{h \perp}\right)\left(\hat{P}_{h \perp} \wedge \vec{p}_{T}\right)}{M M_{h}} h_{1 L}^{\perp} H_{1}^{\perp}\right]\right\}, \\
d^{9} \sigma_{L L}= & \sum_{a} \frac{\alpha^{2} e_{a}^{2}}{2 \pi Q^{2} y}\left|\lambda_{e}\right|\left|S_{L}\right| C(y) \mathcal{I}\left[g_{1 L} D_{1}\right],
\end{aligned}
$$

$$
\begin{aligned}
& d^{9} \sigma_{O T}=\sum_{a} \frac{\alpha^{2} e_{a}^{2}}{2 \pi Q^{2} y}\left|\vec{S}_{T}\right| A(y)\left\{\frac{\left|\vec{R}_{T}\right|}{M_{h}} \sin \left(\phi_{R}-\phi_{S}\right) \mathcal{I}\left[\frac{\vec{p}_{T} \cdot \vec{k}_{T}}{2 M M_{h}} g_{1 T} G_{1}^{\perp}\right]\right. \\
& -\frac{\left|\vec{R}_{T}\right|}{M_{h}} \cos \left(\phi_{R}-\phi_{S}\right) \mathcal{I}\left[\frac{\left(\vec{p}_{T} \cdot \hat{P}_{h \perp}\right)\left(\hat{P}_{h \perp} \wedge \vec{k}_{T}\right)-\left(\vec{k}_{T} \cdot \hat{P}_{h \perp}\right)\left(\hat{P}_{h \perp} \wedge \vec{p}_{T}\right)}{2 M M_{h}} g_{1 T} G_{1}^{\perp}\right] \\
& -\frac{\left|\vec{R}_{T}\right|}{M_{h}} \sin \left(2 \phi_{h}-\phi_{R}-\phi_{S}\right) \mathcal{I}\left[\frac{2\left(\vec{p}_{T} \cdot \hat{P}_{h \perp}\right)\left(\vec{k}_{T} \cdot \hat{P}_{h \perp}\right)-\vec{p}_{T} \cdot \vec{k}_{T}}{2 M M_{h}} g_{1 T} G_{1}^{\perp}\right] \\
& -\frac{\left|\vec{R}_{T}\right|}{M_{h}} \cos \left(2 \phi_{h}-\phi_{R}-\phi_{S}\right) \mathcal{I}\left[\frac{\left(\vec{p}_{T} \cdot \hat{P}_{h \perp}\right)\left(\hat{P}_{h \perp} \wedge \vec{k}_{T}\right)+\left(\vec{k}_{T} \cdot \hat{P}_{h \perp}\right)\left(\hat{P}_{h \perp} \wedge \vec{p}_{T}\right)}{2 M M_{h}} g_{1 T} G_{1}^{\perp}\right] \\
& \left.+\sin \left(\phi_{h}-\phi_{S}\right) \mathcal{I}\left[\frac{\vec{p}_{T} \cdot \hat{P}_{h \perp}}{M} f_{1 T}^{\perp} D_{1}\right]+\cos \left(\phi_{h}-\phi_{S}\right) \mathcal{I}\left[\frac{\hat{P}_{h \perp} \wedge \vec{p}_{T}}{M} f_{1 T}^{\perp} D_{1}\right]\right\} \\
& +\sum_{a} \frac{\alpha^{2} e_{a}^{2}}{2 \pi Q^{2} y}\left|\vec{S}_{T}\right| B(y)\left\{\sin \left(\phi_{h}+\phi_{S}\right) \mathcal{I}\left[\frac{\vec{k}_{T} \cdot \hat{P}_{h \perp}}{M_{h}} h_{1} H_{1}^{\perp}\right]\right. \\
& +\cos \left(\phi_{h}+\phi_{S}\right) \mathcal{I}\left[\frac{\hat{P}_{h \perp} \wedge \vec{k}_{T}}{M_{h}} h_{1} H_{1}^{\perp}\right]+\frac{\left|\vec{R}_{T}\right|}{M_{h}} \sin \left(\phi_{R}+\phi_{S}\right) \mathcal{I}\left[h_{1} \bar{H}_{1}^{\varangle}\right]+\sin \left(3 \phi_{h}-\phi_{S}\right) \\
& \times \mathcal{I}\left[\frac{4\left(\vec{p}_{T} \cdot \hat{P}_{h \perp}\right)^{2}\left(\vec{k}_{T} \cdot \hat{P}_{h \perp}\right)-2\left(\vec{p}_{T} \cdot \hat{P}_{h \perp}\right)\left(\vec{p}_{T} \cdot \vec{k}_{T}\right)-\vec{p}_{T}^{2}\left(\vec{k}_{T} \cdot \hat{P}_{h \perp}\right)}{2 M^{2} M_{h}} h_{1 T}^{\perp} H_{1}^{\perp}\right] \\
& +\cos \left(3 \phi_{h}-\phi_{S}\right) \mathcal{I}\left[\left(\frac{2\left(\vec{p}_{T} \cdot \hat{P}_{h \perp}\right)^{2}\left(\hat{P}_{h \perp} \wedge \vec{k}_{T}\right)+2\left(\vec{k}_{T} \cdot \hat{P}_{h \perp}\right)\left(\vec{p}_{T} \cdot \hat{P}_{h \perp}\right)\left(\hat{P}_{h \perp} \wedge \vec{p}_{T}\right)}{2 M^{2} M_{h}}\right.\right. \\
& \left.\left.-\frac{\vec{p}_{T}^{2}\left(\hat{P}_{h \perp} \wedge \vec{k}_{T}\right)}{2 M^{2} M_{h}}\right) h_{1 T}^{\perp} H_{1}^{\perp}\right]+\frac{\left|\vec{R}_{T}\right|}{M_{h}} \sin \left(2 \phi_{h}+\phi_{R}-\phi_{S}\right) \mathcal{I}\left[\frac{2\left(\vec{p}_{T} \cdot \hat{P}_{h \perp}\right)^{2}-\vec{p}_{T}^{2}}{2 M^{2}} h_{1 T}^{\perp} \bar{H}_{1}^{\varangle}\right] \\
& \left.+\frac{\left|\vec{R}_{T}\right|}{M_{h}} \cos \left(2 \phi_{h}+\phi_{R}-\phi_{S}\right) \mathcal{I}\left[\frac{\left(\vec{p}_{T} \cdot \hat{P}_{h \perp}\right)\left(\hat{P}_{h \perp} \wedge \vec{p}_{T}\right)}{2 M^{2}} h_{1 T}^{\perp} \bar{H}_{1}^{\varangle}\right]\right\},
\end{aligned}
$$




$$
\begin{aligned}
d^{9} \sigma_{L T}= & \sum_{a} \frac{\alpha^{2} e_{a}^{2}}{2 \pi Q^{2} y}\left|\vec{S}_{T}\right| C(y)\left\{\cos \left(\phi_{h}-\phi_{S}\right) \mathcal{I}\left[\frac{\vec{p}_{T} \cdot \hat{P}_{h \perp}}{M} g_{1 T} D_{1}\right]\right. \\
& -\sin \left(\phi_{h}-\phi_{S}\right) \mathcal{I}\left[\frac{\hat{P}_{h \perp} \wedge \vec{p}_{T}}{M} g_{1 T} D_{1}\right] \\
& -\frac{\left|\vec{R}_{T}\right|}{M_{h}} \cos \left(\phi_{R}-\phi_{S}\right) \mathcal{I}\left[\frac{\vec{p}_{T} \cdot \vec{k}_{T}}{2 M M_{h}} f_{1 T}^{\perp} G_{1}^{\perp}\right] \\
& +\frac{\left|\vec{R}_{T}\right|}{M_{h}} \cos \left(2 \phi_{h}-\phi_{R}-\phi_{S}\right) \mathcal{I}\left[\frac{2\left(\vec{p}_{T} \cdot \hat{P}_{h \perp}\right)\left(\vec{k}_{T} \cdot \hat{P}_{h \perp}\right)-\vec{p}_{T} \cdot \vec{k}_{T}}{2 M M_{h}} f_{1 T}^{\perp} G_{1}^{\perp}\right] \\
& -\frac{\left|\vec{R}_{T}\right|}{M_{h}} \sin \left(\phi_{R}-\phi_{S}\right) \mathcal{I}\left[\frac{\left(\vec{p}_{T} \cdot \hat{P}_{h \perp}\right)\left(\hat{P}_{h \perp} \wedge \vec{k}_{T}\right)-\left(\vec{k}_{T} \cdot \hat{P}_{h \perp}\right)\left(\hat{P}_{h \perp} \wedge \vec{p}_{T}\right)}{2 M M_{h}} f_{1 T}^{\perp} G_{1}^{\perp}\right] \\
& \left.+\frac{\left|\vec{R}_{T}\right|}{M_{h}} \sin \left(2 \phi_{h}-\phi_{R}-\phi_{S}\right) \mathcal{I}\left[\frac{\left(\vec{p}_{T} \cdot \hat{P}_{h \perp}\right)\left(\hat{P}_{h \perp} \wedge \vec{k}_{T}\right)+\left(\vec{k}_{T} \cdot \hat{P}_{h \perp}\right)\left(\hat{P}_{h \perp} \wedge \vec{p}_{T}\right)}{2 M M_{h}} f_{1 T}^{\perp} G_{1}^{\perp}\right]\right\} .
\end{aligned}
$$

In the case of $d^{9} \sigma_{O T}$, i.e. for an unpolarized beam and a transversely polarized target, the full expression of the cross section corresponds to the one in Eq.(10) of Ref. [6], apart for a different overall factor, due to slightly different definitions of the hadron tensor and of the fragmentation functions, and the use of $M_{h}$ instead of $M_{1}\left(M_{2}\right)$ in the denominators, due to a different definition of the expansion (15).

\section{APPENDIX B}

The full expression of $\left[\mathcal{P}_{-} \Delta\left(z, \vec{k}_{T}^{2}, M_{h}^{2}\right) \gamma^{-}\right]_{M^{\prime} M \chi_{2}^{\prime} \chi_{2}}^{L^{\prime}}$ in Eq. (58) is

$$
\left[\mathcal{P}_{-} \Delta\left(z, \vec{k}_{T}^{2}, M_{h}^{2}\right) \gamma^{-}\right]_{M^{\prime} M}^{L^{\prime} L} \chi_{2}^{\prime} \chi_{2}=\frac{1}{8}\left(\begin{array}{cc}
A_{M^{\prime} M}^{L^{\prime} L} & B_{M^{\prime} M}^{L^{\prime} L} \\
\left(B_{M^{\prime} M}^{L^{\prime} M}\right)^{\dagger} & C_{M^{\prime} M}^{L^{\prime} M}
\end{array}\right)
$$

where

$$
\begin{aligned}
& A_{M^{\prime} M}^{L^{\prime} L}= \\
& \left(\begin{array}{c|ccc}
D_{1, O O}^{s} & -\sqrt{\frac{2}{3}} e^{i \phi}\left(D_{1, O T}+i \frac{\left|\vec{k}_{T}\right||\vec{R}|}{M_{h}^{2}} G_{1, O T}^{\perp}\right) & \frac{2}{\sqrt{3}} D_{1, O L} & \sqrt{\frac{2}{3}} e^{-i \phi}\left(D_{1, O T}-i \frac{\left|\vec{k}_{T}\right||\vec{R}|}{M_{h}^{2}} G_{1, O T}^{\perp}\right) \\
\hline-\sqrt{\frac{2}{3}} e^{-i \phi}\left(D_{1, O T}-i \frac{\left|\vec{k}_{T}\right||\vec{R}|}{M_{h}^{2}} G_{1, O T}^{\perp}\right) & D_{1, O O}^{p}-\frac{1}{3} D_{1, L L} & -\frac{\sqrt{2}}{3} e^{-i \phi}\left(D_{1, L T}-i \frac{\left|\vec{k}_{T}\right||\vec{R}|}{M_{h}^{2}} G_{1, L T}^{\perp}\right) & -\frac{2}{3} e^{-2 i \phi}\left(2 D_{1, T T}-i \frac{\left|\vec{k}_{T}\right||\vec{R}|}{M_{h}^{2}} G_{1, T T}^{\perp}\right) \\
\frac{2}{\sqrt{3}} D_{1, O L} & -\frac{\sqrt{2}}{3} e^{i \phi}\left(D_{1, L T}+i \frac{\left|\vec{k}_{T}\right||\vec{R}|}{M_{h}^{2}} G_{1, L T}^{\perp}\right) & D_{1, O O}^{p}+\frac{2}{3} D_{1, L L} & \frac{\sqrt{2}}{3} e^{-i \phi}\left(D_{1, L T}-i \frac{\left|\vec{k}_{T}\right||\vec{R}|}{M_{h}^{2}} G_{1, L T}^{\perp}\right) \\
\sqrt{\frac{2}{3}} e^{i \phi}\left(D_{1, O T}+i \frac{\left|\vec{k}_{T}\right||\vec{R}|}{M_{h}^{2}} G_{1, O T}^{\perp}\right) & -\frac{2}{3} e^{2 i \phi}\left(2 D_{1, T T}+i \frac{\left|\vec{k}_{T}\right||\vec{R}|}{M_{h}^{2}} G_{1, T T}^{\perp}\right) & \frac{\sqrt{2}}{3} e^{i \phi}\left(D_{1, L T}+i \frac{\left|\vec{k}_{T}\right||\vec{R}|}{M_{h}^{2}} G_{1, L T}^{\perp}\right) & D_{1, O O}^{p}-\frac{1}{3} D_{1, L L}
\end{array}\right)
\end{aligned}
$$

$$
\begin{aligned}
& B_{M^{\prime} M}^{L^{\prime} L}= \\
& i \frac{\left|\vec{k}_{T}\right|}{M_{h}}\left(\begin{array}{c|ccc}
e^{i \phi} H_{1, O O}^{\perp} & -\frac{2 \sqrt{2}}{\sqrt{3}} e^{2 i \phi} H_{1, O T}^{\perp} & \frac{2}{\sqrt{3}} e^{i \phi} H_{1, O L}^{\perp} & \frac{2 \sqrt{2}}{\sqrt{3}}\left(\frac{|\vec{k}|}{|\vec{k} T|} \bar{H}_{1, O T}^{\searrow}+H_{1, O T}^{\perp}\right) \\
\hline-\frac{2 \sqrt{2}}{\sqrt{3}}\left(\frac{|\vec{k}|}{|\vec{k} T|} \bar{H}_{1, O T}^{\searrow}+H_{1, O T}^{\perp}\right) & e^{i \phi}\left(H_{1, O O}^{\perp p}-\frac{1}{3} H_{1, L L}^{\perp}\right) & -\frac{2 \sqrt{2}}{3}\left(\frac{|\vec{k}|}{|\vec{k} T|} \bar{H}_{1, L T}^{\searrow}+H_{1, L T}^{\perp}\right) & -\frac{8}{3} e^{i \phi}\left(\frac{|\vec{k}|}{\mid \vec{k}} H_{1, T T}^{\searrow}+H_{1, T T}^{\perp}\right) \\
\frac{2}{\sqrt{3}} e^{i \phi} H_{1, O L}^{\perp} & -\frac{2 \sqrt{2}}{\sqrt{3}} e^{2 i \phi} H_{1, T T}^{\perp} & e^{i \phi}\left(H_{1, O O}^{\perp}+\frac{2}{3} H_{1, L L}^{\perp}\right) & \frac{2 \sqrt{2}}{3}\left(\frac{|\vec{R}|}{|\vec{k} T|} \bar{H}_{1, L T}^{\searrow}+H_{1, L T}^{\perp}\right) \\
\frac{2 \sqrt{2}}{\sqrt{3}} e^{2 i \phi} H_{1, O T}^{\perp} & -\frac{8}{3} e^{3 i \phi} H_{1, T T}^{\perp} & \frac{2 \sqrt{2}}{\sqrt{3}} e^{2 i \phi} H_{1, T T}^{\perp} & e^{i \phi}\left(H_{1, O O}^{\perp}-\frac{1}{3} H_{1, L L}^{\perp}\right)
\end{array}\right),
\end{aligned}
$$

and $\phi \equiv \phi_{k}-\phi_{R}$. The matrix (B1) respects Hermiticity, angular momentum conservation, and parity invariance. Due to the explicit dependence upon the transverse momentum $\vec{k}_{T}$, the conditions for angular momentum and parity conservation read

$$
\begin{aligned}
M+\chi_{2}^{\prime} & =M^{\prime}+\chi_{2}+l_{k_{T}} \\
{\left[\mathcal{P}_{-} \Delta \gamma^{-}\right]_{M^{\prime} M \chi_{2}^{\prime} \chi_{2}}^{L^{\prime} L} } & =(-1)^{l_{k_{T}}}\left[\mathcal{P}_{-} \Delta \gamma^{-}\right]_{-M^{\prime}-M-\chi_{2}^{\prime}-\chi_{2}}^{L^{\prime} L}
\end{aligned}
$$


where $l_{k_{T}}$ denotes the units of angular momentum introduced by $\vec{k}_{T}$. From the last constraint it is possible to derive the lower right block, i.e. $C_{M^{\prime} M}^{L^{\prime} L}=(-1)^{l_{k}} A_{-M^{\prime}-M}^{L^{\prime} L}$.

Again, as in the case of Eq. (39), we have

$$
H_{1, O O}\left(z, \vec{k}_{T}^{2}, M_{h}^{2}\right)=\frac{1}{4} H_{1, O O}^{\perp s}\left(z, \vec{k}_{T}^{2}, M_{h}^{2}\right)+\frac{3}{4} H_{1, O O}^{\perp p}\left(z, \vec{k}_{T}^{2}, M_{h}^{2}\right)
$$

and the functions $H_{1, O O}^{\perp s}, H_{1, O O}^{\perp p}$ are kinematically indistinguishable unless some hypothesis is made on their $M_{h}^{2}$ dependence. The $L=L^{\prime}=1$ sector of Eqs. (B2, B3) has been studied in the case of spin-1 fragmentation [13]. The interference $\left(L=0, L^{\prime}=1\right)$ sector has never been analyzed in this form, namely including the explicit dependence on $\vec{k}_{T}$. Finally, from $\left[\mathcal{P}_{-} \Delta \gamma^{-}\right]_{M^{\prime} M \chi_{2}^{\prime} \chi_{2}}^{L^{\prime}}$ being positive semidefinite, it is possible to derive bounds on each of the displayed fragmentation functions.

\section{APPENDIX C}

In this appendix, we explicitly present the complete cross section for the production of two unpolarized hadrons in relative $s$ and $p$ waves, at leading order in $1 / Q$, including transverse momenta and T-odd distribution and fragmentation functions.

The cross section is obtained by replacing Eqs. (12,51,22, B1, 34) in Eq. (59). It is convenient to introduce the following combination of fragmentation functions

$$
\begin{aligned}
& H_{1, O T}^{\varangle}=\bar{H}_{1, O T}^{\varangle}+\frac{\left|\vec{k}_{T}\right|}{|\vec{R}|} H_{1, O T}^{\perp}, \\
& H_{1, L T}^{\varangle}=\bar{H}_{1, L T}^{\varangle}+\frac{\left|\vec{k}_{T}\right|}{|\vec{R}|} H_{1, L T}^{\perp}, \\
& H_{1, T T}^{\varangle}=\bar{H}_{1, T T}^{\varangle}+\frac{\left|\vec{k}_{T}\right|}{|\vec{R}|} H_{1, T T}^{\perp} .
\end{aligned}
$$


a. Unpolarized lepton beam and unpolarized target

$$
\begin{aligned}
& d^{8} \sigma_{O O}=\sum_{a} \frac{\alpha^{2} e_{a}^{2}}{2 \pi s x y^{2}} A(y)\left\{\mathcal{I}\left[f_{1}\left(\frac{1}{4} D_{1, O O}^{s}+\frac{3}{4} D_{1, O O}^{p}\right)\right]+\cos \theta \mathcal{I}\left[f_{1} D_{1, O L}\right]\right. \\
& +\frac{1}{3}\left(3 \cos ^{2} \theta-1\right) \mathcal{I}\left[f_{1}\left(\frac{3}{4} D_{1, L L}\right)\right]+\sin \theta \cos \left(\phi_{h}-\phi_{R}\right) \mathcal{I}\left[\frac{\vec{k}_{T} \cdot \hat{P}_{h \perp}}{M_{h}} f_{1}\left(-\frac{M_{h}}{\left|\vec{k}_{T}\right|} D_{1, O T}\right)\right] \\
& -\sin 2 \theta \cos \left(\phi_{h}-\phi_{R}\right) \mathcal{I}\left[\frac{\vec{k}_{T} \cdot \hat{P}_{h \perp}}{M_{h}} f_{1}\left(-\frac{M_{h}}{2\left|\vec{k}_{T}\right|} D_{1, L T}\right)\right]-\sin ^{2} \theta \cos \left(2 \phi_{h}-2 \phi_{R}\right) \\
& \left.\times \mathcal{I}\left[\frac{2\left(\vec{k}_{T} \cdot \hat{P}_{h \perp}\right)^{2}-\vec{k}_{T}^{2}}{M_{h}^{2}} f_{1}\left(-\frac{M_{h}^{2}}{\left|\vec{k}_{T}\right|^{2}} D_{1, T T}\right)\right]\right\} \\
& +\sum_{a} \frac{\alpha^{2} e_{a}^{2}}{2 \pi s x y^{2}} B(y)\left\{-\cos 2 \phi_{h} \mathcal{I}\left[\frac{2\left(\vec{p}_{T} \cdot \hat{P}_{h \perp}\right)\left(\vec{k}_{T} \cdot \hat{P}_{h \perp}\right)-\vec{p}_{T} \cdot \vec{k}_{T}}{M M_{h}} h_{1}^{\perp}\left(\frac{1}{4} H_{1, O O}^{\perp s}+\frac{3}{4} H_{1, O O}^{\perp p}\right)\right]\right. \\
& -\frac{1}{3}\left(3 \cos ^{2} \theta-1\right) \cos 2 \phi_{h} \mathcal{I}\left[\frac{2\left(\vec{p}_{T} \cdot \hat{P}_{h \perp}\right)\left(\vec{k}_{T} \cdot \hat{P}_{h \perp}\right)-\vec{p}_{T} \cdot \vec{k}_{T}}{M M_{h}} h_{1}^{\perp}\left(\frac{3}{4} H_{1, L L}^{\perp}\right)\right] \\
& +\sin \theta \cos \left(\phi_{h}+\phi_{R}\right) \mathcal{I}\left[\frac{\vec{p}_{T} \cdot \hat{P}_{h \perp}}{M} h_{1}^{\perp}\left(-\frac{|\vec{R}|}{M_{h}} H_{1, O T}^{\varangle}\right)\right]+\sin 2 \theta \cos \left(\phi_{h}+\phi_{R}\right) \\
& \times \mathcal{I}\left[\frac{\vec{p}_{T} \cdot \hat{P}_{h \perp}}{M} h_{1}^{\perp}\left(-\frac{|\vec{R}|}{2 M_{h}} H_{1, L T}^{\varangle}\right)\right]+\sin ^{2} \theta \cos 2 \phi_{R} \mathcal{I}\left[\frac{\vec{p}_{T} \cdot \vec{k}_{T}}{M M_{h}} h_{1}^{\perp}\left(-\frac{|\vec{R}|}{\left|\vec{k}_{T}\right|} H_{1, T T}^{\varangle}\right)\right] \\
& +\sin \theta \cos \left(3 \phi_{h}-\phi_{R}\right) \\
& \times \mathcal{I}\left[\frac{4\left(\vec{k}_{T} \cdot \hat{P}_{h \perp}\right)^{2}\left(\vec{p}_{T} \cdot \hat{P}_{h \perp}\right)-2\left(\vec{k}_{T} \cdot \hat{P}_{h \perp}\right)\left(\vec{p}_{T} \cdot \vec{k}_{T}\right)-\vec{k}_{T}^{2}\left(\vec{p}_{T} \cdot \hat{P}_{h \perp}\right)}{2 M M_{h}^{2}} h_{1}^{\perp}\left(-\frac{2 M_{h}}{\left|\vec{k}_{T}\right|} H_{1, O T}^{\perp}\right)\right] \\
& +\sin 2 \theta \cos \left(3 \phi_{h}-\phi_{R}\right) \\
& \times \mathcal{I}\left[\frac{4\left(\vec{k}_{T} \cdot \hat{P}_{h \perp}\right)^{2}\left(\vec{p}_{T} \cdot \hat{P}_{h \perp}\right)-2\left(\vec{k}_{T} \cdot \hat{P}_{h \perp}\right)\left(\vec{p}_{T} \cdot \vec{k}_{T}\right)-\vec{k}_{T}^{2}\left(\vec{p}_{T} \cdot \hat{P}_{h \perp}\right)}{2 M M_{h}^{2}} h_{1}^{\perp}\left(-\frac{M_{h}}{\left|\vec{k}_{T}\right|} H_{1, L T}^{\perp}\right)\right] \\
& +\sin ^{2} \theta \cos \left(4 \phi_{h}-2 \phi_{R}\right) \mathcal{I}\left[\left(\frac{\left[\vec{k}_{T}^{2}-4\left(\vec{k}_{T} \cdot \hat{P}_{h \perp}\right)^{2}\right]\left[\vec{p}_{T} \cdot \vec{k}_{T}-4\left(\vec{k}_{T} \cdot \hat{P}_{h \perp}\right)\left(\vec{p}_{T} \cdot \hat{P}_{h \perp}\right)\right]}{2 M M_{h}^{3}}\right.\right. \\
& \left.\left.\left.-\frac{8\left(\vec{k}_{T} \cdot \hat{P}_{h \perp}\right)^{3}\left(\vec{p}_{T} \cdot \hat{P}_{h \perp}\right)}{2 M M_{h}^{3}}\right) h_{1}^{\perp}\left(-\frac{2 M_{h}^{2}}{\left|\vec{k}_{T}\right|^{2}} H_{1, T T}^{\perp}\right)\right]\right\}
\end{aligned}
$$

b. Polarized lepton beam and unpolarized target

$$
\begin{aligned}
d^{8} \sigma_{L O}= & -\sum_{a} \frac{\alpha^{2} e_{a}^{2}}{2 \pi s x y^{2}} \lambda_{e} C(y)\left\{\sin \theta \sin \left(\phi_{h}-\phi_{R}\right) \mathcal{I}\left[\frac{\vec{k}_{T} \cdot \hat{P}_{h \perp}}{M_{h}} f_{1}\left(\frac{|\vec{R}|}{M_{h}} G_{1, O T}^{\perp}\right)\right]\right. \\
& +\sin 2 \theta \sin \left(\phi_{h}-\phi_{R}\right) \mathcal{I}\left[\frac{\vec{k}_{T} \cdot \hat{P}_{h \perp}}{M_{h}} f_{1}\left(\frac{|\vec{R}|}{2 M_{h}} G_{1, L T}^{\perp}\right)\right] \\
& \left.+\sin ^{2} \theta \sin \left(2 \phi_{h}-2 \phi_{R}\right) \mathcal{I}\left[\frac{2\left(\vec{k}_{T} \cdot \hat{P}_{h \perp}\right)^{2}-\vec{k}_{T}^{2}}{M_{h}^{2}} f_{1}\left(\frac{|\vec{R}|}{2\left|\vec{k}_{T}\right|} G_{1, T T}^{\perp}\right)\right]\right\}
\end{aligned}
$$


c. Unpolarized lepton beam and longitudinally polarized target

$$
\begin{aligned}
& d^{8} \sigma_{O L}=-\sum_{a} \frac{\alpha^{2} e_{a}^{2}}{2 \pi s x y^{2}}\left|S_{L}\right| A(y)\left\{\sin \theta \sin \left(\phi_{h}-\phi_{R}\right) \mathcal{I}\left[\frac{\vec{k}_{T} \cdot \hat{P}_{h \perp}}{M_{h}} g_{1 L}\left(\frac{|\vec{R}|}{M_{h}} G_{1, O T}^{\perp}\right)\right]\right. \\
& +\sin 2 \theta \sin \left(\phi_{h}-\phi_{R}\right) \mathcal{I}\left[\frac{\vec{k}_{T} \cdot \hat{P}_{h \perp}}{M_{h}} g_{1 L}\left(\frac{|\vec{R}|}{2 M_{h}} G_{1, L T}^{\perp}\right)\right] \\
& \left.+\sin ^{2} \theta \sin \left(2 \phi_{h}-2 \phi_{R}\right) \mathcal{I}\left[\frac{2\left(\vec{k}_{T} \cdot \hat{P}_{h \perp}\right)^{2}-\vec{k}_{T}^{2}}{M_{h}^{2}} g_{1 L}\left(\frac{|\vec{R}|}{2\left|\vec{k}_{T}\right|} G_{1, T T}^{\perp}\right)\right]\right\} \\
& -\sum_{a} \frac{\alpha^{2} e_{a}^{2}}{2 \pi s x y^{2}}\left|S_{L}\right| B(y)\left\{\sin 2 \phi_{h} \mathcal{I}\left[\frac{2\left(\vec{p}_{T} \cdot \hat{P}_{h \perp}\right)\left(\vec{k}_{T} \cdot \hat{P}_{h \perp}\right)-\vec{p}_{T} \cdot \vec{k}_{T}}{M M_{h}} h_{1 L}^{\perp}\left(\frac{1}{4} H_{1, O O}^{\perp s}+\frac{3}{4} H_{1, O O}^{\perp p}\right)\right]\right. \\
& -\frac{1}{3}\left(3 \cos ^{2} \theta-1\right) \sin 2 \phi_{h} \times \mathcal{I}\left[\frac{2\left(\vec{p}_{T} \cdot \hat{P}_{h \perp}\right)\left(\vec{k}_{T} \cdot \hat{P}_{h \perp}\right)-\vec{p}_{T} \cdot \vec{k}_{T}}{M M_{h}} h_{1 L}^{\perp}\left(\frac{3}{4} H_{1, L L}^{\perp}\right)\right] \\
& +\sin \theta \sin \left(\phi_{h}+\phi_{R}\right) \mathcal{I}\left[\frac{\vec{p}_{T} \cdot \hat{P}_{h \perp}}{M} h_{1 L}^{\perp}\left(-\frac{|\vec{R}|}{M_{h}} H_{1, O T}^{\varangle}\right)\right]+\sin 2 \theta \sin \left(\phi_{h}+\phi_{R}\right) \\
& \times \mathcal{I}\left[\frac{\vec{p}_{T} \cdot \hat{P}_{h \perp}}{M} h_{1 L}^{\perp}\left(-\frac{|\vec{R}|}{2 M_{h}} H_{1, L T}^{\varangle}\right)\right]+\sin ^{2} \theta \sin 2 \phi_{R} \mathcal{I}\left[\frac{\vec{p}_{T} \cdot \vec{k}_{T}}{M M_{h}} h_{1 L}^{\perp}\left(-\frac{|\vec{R}|}{\left|\vec{k}_{T}\right|} H_{1, T T}^{\varangle}\right)\right] \\
& +\sin \theta \sin \left(3 \phi_{h}-\phi_{R}\right) \\
& \times \mathcal{I}\left[\frac{4\left(\vec{k}_{T} \cdot \hat{P}_{h \perp}\right)^{2}\left(\vec{p}_{T} \cdot \hat{P}_{h \perp}\right)-2\left(\vec{k}_{T} \cdot \hat{P}_{h \perp}\right)\left(\vec{p}_{T} \cdot \vec{k}_{T}\right)-\vec{k}_{T}^{2}\left(\vec{p}_{T} \cdot \hat{P}_{h \perp}\right)}{2 M M_{h}^{2}} h_{1 L}^{\perp}\left(-\frac{2 M_{h}}{\left|\vec{k}_{T}\right|} H_{1, O T}^{\perp}\right)\right] \\
& +\sin 2 \theta \sin \left(3 \phi_{h}-\phi_{R}\right) \\
& \times \mathcal{I}\left[\frac{4\left(\vec{k}_{T} \cdot \hat{P}_{h \perp}\right)^{2}\left(\vec{p}_{T} \cdot \hat{P}_{h \perp}\right)-2\left(\vec{k}_{T} \cdot \hat{P}_{h \perp}\right)\left(\vec{p}_{T} \cdot \vec{k}_{T}\right)-\vec{k}_{T}^{2}\left(\vec{p}_{T} \cdot \hat{P}_{h \perp}\right)}{2 M M_{h}^{2}} h_{1 L}^{\perp}\left(-\frac{M_{h}}{\left|\vec{k}_{T}\right|} H_{1, L T}^{\perp}\right)\right] \\
& +\sin ^{2} \theta \sin \left(4 \phi_{h}-2 \phi_{R}\right) \mathcal{I}\left[\left(\frac{\left[\vec{k}_{T}^{2}-4\left(\vec{k}_{T} \cdot \hat{P}_{h \perp}\right)^{2}\right]\left[\vec{p}_{T} \cdot \vec{k}_{T}-4\left(\vec{k}_{T} \cdot \hat{P}_{h \perp}\right)\left(\vec{p}_{T} \cdot \hat{P}_{h \perp}\right)\right]}{2 M M_{h}^{3}}\right.\right. \\
& \left.\left.\left.-\frac{8\left(\vec{k}_{T} \cdot \hat{P}_{h \perp}\right)^{3}\left(\vec{p}_{T} \cdot \hat{P}_{h \perp}\right)}{2 M M_{h}^{3}}\right) h_{1 L}^{\perp}\left(-\frac{2 M_{h}^{2}}{\left|\vec{k}_{T}\right|^{2}} H_{1, T T}^{\perp}\right)\right]\right\}
\end{aligned}
$$

d. Polarized lepton beam and longitudinally polarized target

$$
\begin{aligned}
d^{8} \sigma_{L L}= & \sum_{a} \frac{\alpha^{2} e_{a}^{2}}{2 \pi s x y^{2}} \lambda_{e}\left|S_{L}\right| C(y)\left\{\mathcal{I}\left[g_{1 L}\left(\frac{1}{4} D_{1, O O}^{s}+\frac{3}{4} D_{1, O O}^{p}\right)\right]+\cos \theta \mathcal{I}\left[g_{1 L} D_{1, O L}\right]\right. \\
& +\frac{1}{3}\left(3 \cos ^{2} \theta-1\right) \mathcal{I}\left[g_{1 L}\left(\frac{3}{4} D_{1, L L}\right)\right] \\
& -\sin \theta \cos \left(\phi_{h}-\phi_{R}\right) \mathcal{I}\left[\frac{\vec{k}_{T} \cdot \hat{P}_{h \perp}}{M_{h}} g_{1 L}\left(-\frac{M_{h}}{\left|\vec{k}_{T}\right|} D_{1, L T}\right)\right] \\
& -\sin 2 \theta \cos \left(\phi_{h}-\phi_{R}\right) \mathcal{I}\left[\frac{\vec{k}_{T} \cdot \hat{P}_{h \perp}}{M_{h}} g_{1 L}\left(-\frac{M_{h}}{2\left|\vec{k}_{T}\right|} D_{1, L T}\right)\right] \\
& \left.-\sin ^{2} \theta \cos \left(2 \phi_{h}-2 \phi_{R}\right) \mathcal{I}\left[\frac{2\left(\vec{k}_{T} \cdot \hat{P}_{h \perp}\right)^{2}-\vec{k}_{T}^{2}}{M_{h}^{2}} g_{1 L}\left(-\frac{M_{h}^{2}}{\left|\vec{k}_{T}\right|^{2}} D_{1, T T}\right)\right]\right\}
\end{aligned}
$$


e. Unpolarized lepton beam and transversely polarized target

$$
\begin{aligned}
& d^{8} \sigma_{O T}=\sum_{a} \frac{\alpha^{2} e_{a}^{2}}{2 \pi s x y^{2}}\left|\vec{S}_{T}\right| A(y)\left\{\sin \theta \sin \left(\phi_{R}-\phi_{S}\right) \mathcal{I}\left[\frac{\left(\vec{p}_{T} \cdot \vec{k}_{T}\right)}{2 M M_{h}} g_{1 T}\left(\frac{|\vec{R}|}{M_{h}} G_{1, O T}^{\perp}\right)\right]\right. \\
& -\sin \theta \sin \left(2 \phi_{h}-\phi_{R}-\phi_{S}\right) \mathcal{I}\left[\frac{2\left(\vec{p}_{T} \cdot \hat{P}_{h \perp}\right)\left(\vec{k}_{T} \cdot \hat{P}_{h \perp}\right)-\vec{p}_{T} \cdot \vec{k}_{T}}{2 M M_{h}} g_{1 T}\left(\frac{|\vec{R}|}{M_{h}} G_{1, O T}^{\perp}\right)\right] \\
& +\sin 2 \theta \sin \left(\phi_{R}-\phi_{S}\right) \mathcal{I}\left[\frac{\left(\vec{p}_{T} \cdot \vec{k}_{T}\right)}{2 M M_{h}} g_{1 T}\left(\frac{|\vec{R}|}{2 M_{h}} G_{1, L T}^{\perp}\right)\right] \\
& -\sin 2 \theta \sin \left(2 \phi_{h}-\phi_{R}-\phi_{S}\right) \mathcal{I}\left[\frac{2\left(\vec{p}_{T} \cdot \hat{P}_{h \perp}\right)\left(\vec{k}_{T} \cdot \hat{P}_{h \perp}\right)-\vec{p}_{T} \cdot \vec{k}_{T}}{2 M M_{h}} g_{1 T}\left(\frac{|\vec{R}|}{2 M_{h}} G_{1, L T}^{\perp}\right)\right] \\
& -\sin ^{2} \theta \sin \left(\phi_{h}-2 \phi_{R}+\phi_{S}\right) \mathcal{I}\left[\frac{2\left(\vec{k}_{T} \cdot \hat{P}_{h \perp}\right)\left(\vec{p}_{T} \cdot \vec{k}_{T}\right)-\vec{k}_{T}^{2}\left(\vec{p}_{T} \cdot \hat{P}_{h \perp}\right)}{2 M M_{h}^{2}} g_{1 T}\left(\frac{\mid \vec{R}}{2\left|\vec{k}_{T}\right|} G_{1, T T}^{\perp}\right)\right] \\
& -\sin ^{2} \theta \sin \left(3 \phi_{h}-2 \phi_{R}-\phi_{S}\right) \mathcal{I}\left[\left(\frac{4\left(\vec{k}_{T} \cdot \hat{P}_{h \perp}\right)^{2}\left(\vec{p}_{T} \cdot \hat{P}_{h \perp}\right)-2\left(\vec{k}_{T} \cdot \hat{P}_{h \perp}\right)\left(\vec{p}_{T} \cdot \vec{k}_{T}\right)}{2 M M_{h}^{2}}\right.\right. \\
& \left.\left.-\frac{\vec{k}_{T}^{2}\left(\vec{p}_{T} \cdot \hat{P}_{h \perp}\right)}{2 M M_{h}^{2}}\right) g_{1 T}\left(\frac{|\vec{R}|}{2\left|\vec{k}_{T}\right|} G_{1, T T}^{\perp}\right)\right]+\sin \left(\phi_{h}-\phi_{S}\right) \mathcal{I}\left[\frac{\vec{p}_{T} \cdot \hat{P}_{h \perp}}{M} f_{1 T}^{\perp}\left(\frac{1}{4} D_{1, O O}^{s}+\frac{3}{4} D_{1, O O}^{p}\right)\right] \\
& +\cos \theta \mathcal{I}\left[f_{1 T}^{\perp} D_{1, O L}\right]+\frac{1}{3}\left(3 \cos ^{2} \theta-1\right) \sin \left(\phi_{h}-\phi_{S}\right) \mathcal{I}\left[\frac{\vec{p}_{T} \cdot \hat{P}_{h \perp}}{M} f_{1 T}^{\perp}\left(\frac{3}{4} D_{1, L L}\right)\right] \\
& -\sin \theta \sin \left(\phi_{R}-\phi_{S}\right) \mathcal{I}\left[\frac{\left(\vec{p}_{T} \cdot \vec{k}_{T}\right)}{2 M M_{h}} f_{1 T}^{\perp}\left(-\frac{M_{h}}{\left|\vec{k}_{T}\right|} D_{1, O T}\right)\right] \\
& -\sin \theta \sin \left(2 \phi_{h}-\phi_{R}-\phi_{S}\right) \mathcal{I}\left[\frac{2\left(\vec{p}_{T} \cdot \hat{P}_{h \perp}\right)\left(\vec{k}_{T} \cdot \hat{P}_{h \perp}\right)-\vec{p}_{T} \cdot \vec{k}_{T}}{2 M M_{h}} f_{1 T}^{\perp}\left(-\frac{M_{h}}{\left|\vec{k}_{T}\right|} D_{1, O T}\right)\right] \\
& -\sin 2 \theta \sin \left(\phi_{R}-\phi_{S}\right) \mathcal{I}\left[\frac{\left(\vec{p}_{T} \cdot \vec{k}_{T}\right)}{2 M M_{h}} f_{1 T}^{\perp}\left(-\frac{M_{h}}{2\left|\vec{k}_{T}\right|} D_{1, L T}\right)\right] \\
& -\sin 2 \theta \sin \left(2 \phi_{h}-\phi_{R}-\phi_{S}\right) \mathcal{I}\left[\frac{2\left(\vec{p}_{T} \cdot \hat{P}_{h \perp}\right)\left(\vec{k}_{T} \cdot \hat{P}_{h \perp}\right)-\vec{p}_{T} \cdot \vec{k}_{T}}{2 M M_{h}} f_{1 T}^{\perp}\left(-\frac{M_{h}}{2\left|\vec{k}_{T}\right|} D_{1, L T}\right)\right] \\
& +\sin ^{2} \theta \sin \left(\phi_{h}-2 \phi_{R}+\phi_{S}\right) \mathcal{I}\left[\frac{2\left(\vec{k}_{T} \cdot \hat{P}_{h \perp}\right)\left(\vec{p}_{T} \cdot \vec{k}_{T}\right)-\vec{k}_{T}^{2}\left(\vec{p}_{T} \cdot \hat{P}_{h \perp}\right)}{2 M M_{h}^{2}} f_{1 T}^{\perp}\left(-\frac{M_{h}^{2}}{\left|\vec{k}_{T}\right|^{2}} D_{1, T T}\right)\right] \\
& -\sin ^{2} \theta \sin \left(3 \phi_{h}-2 \phi_{R}-\phi_{S}\right) \mathcal{I}\left[\left(\frac{4\left(\vec{k}_{T} \cdot \hat{P}_{h \perp}\right)^{2}\left(\vec{p}_{T} \cdot \hat{P}_{h \perp}\right)-2\left(\vec{k}_{T} \cdot \hat{P}_{h \perp}\right)\left(\vec{p}_{T} \cdot \vec{k}_{T}\right)}{2 M M_{h}^{2}}\right.\right. \\
& \left.\left.\left.-\frac{\vec{k}_{T}^{2}\left(\vec{p}_{T} \cdot \hat{P}_{h \perp}\right)}{2 M M_{h}^{2}}\right) f_{1 T}^{\perp}\left(-\frac{M_{h}^{2}}{\left|\vec{k}_{T}\right|^{2}} D_{1, T T}\right)\right]\right\} \\
& +\sum_{a} \frac{\alpha^{2} e_{a}^{2}}{2 \pi s x y^{2}} B(y)\left\{\cos 2 \phi_{h} \mathcal{I}\left[\frac{2\left(\vec{p}_{T} \cdot \hat{P}_{h \perp}\right)\left(\vec{k}_{T} \cdot \hat{P}_{h \perp}\right)-\vec{p}_{T} \cdot \vec{k}_{T}}{M M_{h}} h_{1}\left(\frac{1}{4} H_{1, O O}^{\perp s}+\frac{3}{4} H_{1, O O}^{\perp p}\right)\right]\right. \\
& +\frac{1}{3}\left(3 \cos ^{2} \theta-1\right) \sin \left(\phi_{h}+\phi_{S}\right) \mathcal{I}\left[\frac{\vec{k}_{T} \cdot \hat{P}_{h \perp}}{M_{h}} h_{1}\left(\frac{3}{4} H_{1, L L}^{\perp}\right)\right] \\
& -\sin \theta \sin \left(\phi_{R}+\phi_{S}\right) \mathcal{I}\left[h_{1}\left(-\frac{|\vec{R}|}{M_{h}} H_{1, \text { OT }}^{\varangle}\right)\right]
\end{aligned}
$$




$$
\begin{aligned}
& -\sin 2 \theta \sin \left(\phi_{R}+\phi_{S}\right) \mathcal{I}\left[h_{1}\left(-\frac{|\vec{R}|}{2 M_{h}} H_{1, L T}^{\varangle}\right)\right] \\
& +\sin ^{2} \theta \sin \left(\phi_{h}-2 \phi_{R}-\phi_{S}\right) \mathcal{I}\left[\frac{\vec{k}_{T} \cdot \hat{P}_{h \perp}}{M_{h}} h_{1}\left(-\frac{|\vec{R}|}{\left|\vec{k}_{T}\right|} H_{1, T T}^{\varangle}\right)\right] \\
& -\sin \theta \sin \left(2 \phi_{h}-\phi_{R}+\phi_{S}\right) \mathcal{I}\left[\frac{2\left(\vec{k}_{T} \cdot \hat{P}_{h \perp}\right)^{2}-\vec{k}_{T}^{2}}{2 M_{h}^{2}} h_{1}\left(-\frac{2 M_{h}}{\left|\vec{k}_{T}\right|} H_{1, O T}^{\perp}\right)\right] \\
& -\sin 2 \theta \sin \left(2 \phi_{h}-\phi_{R}+\phi_{S}\right) \mathcal{I}\left[\frac{2\left(\vec{k}_{T} \cdot \hat{P}_{h \perp}\right)^{2}-\vec{k}_{T}^{2}}{2 M_{h}^{2}} h_{1}\left(-\frac{M_{h}}{\left|\vec{k}_{T}\right|} H_{1, L T}^{\perp}\right)\right] \\
& -\sin ^{2} \theta \sin \left(3 \phi_{h}-2 \phi_{R}+\phi_{S}\right) \mathcal{I}\left[\frac{4\left(\vec{k}_{T} \cdot \hat{P}_{h \perp}\right)^{3}-3 \vec{k}_{T}^{2}\left(\vec{k}_{T} \cdot \hat{P}_{h \perp}\right)}{2 M_{h}^{3}} h_{1}\left(-\frac{2 M_{h}^{2}}{\left|\vec{k}_{T}\right|^{2}} H_{1, T T}^{\perp}\right)\right] \\
& +\cos 2 \phi_{h} \mathcal{I}\left[\frac{2\left(\vec{p}_{T} \cdot \hat{P}_{h \perp}\right)\left(\vec{k}_{T} \cdot \hat{P}_{h \perp}\right)-\vec{p}_{T} \cdot \vec{k}_{T}}{M M_{h}} h_{1 T}^{\perp}\left(\frac{1}{4} H_{1, O O}^{\perp s}+\frac{3}{4} H_{1, O O}^{\perp p}\right)\right] \\
& +\frac{1}{3}\left(3 \cos ^{2} \theta-1\right) \sin \left(3 \phi_{h}-\phi_{S}\right) \\
& \times \mathcal{I}\left[\frac{4\left(\vec{p}_{T} \cdot \hat{P}_{h \perp}\right)^{2}\left(\vec{k}_{T} \cdot \hat{P}_{h \perp}\right)-2\left(\vec{p}_{T} \cdot \hat{P}_{h \perp}\right)\left(\vec{p}_{T} \cdot \vec{k}_{T}\right)-\vec{p}_{T}^{2}\left(\vec{k}_{T} \cdot \hat{P}_{h \perp}\right)}{2 M^{2} M_{h}} h_{1 T}^{\perp}\left(\frac{3}{4} H_{1, L L}^{\perp}\right)\right] \\
& -\sin \theta \sin \left(2 \phi_{h}+\phi_{R}-\phi_{S}\right) \mathcal{I}\left[\frac{2\left(\vec{p}_{T} \cdot \hat{P}_{h \perp}\right)^{2}-\vec{p}_{T}^{2}}{2 M^{2}} h_{1 T}^{\perp}\left(-\frac{|\vec{R}|}{M_{h}} H_{1, O T}^{\varangle}\right)\right] \\
& -\sin 2 \theta \sin \left(2 \phi_{h}+\phi_{R}-\phi_{S}\right) \mathcal{I}\left[\frac{2\left(\vec{p}_{T} \cdot \hat{P}_{h \perp}\right)^{2}-\vec{p}_{T}^{2}}{2 M^{2}} h_{1 T}^{\perp}\left(-\frac{|\vec{R}|}{2 M_{h}} H_{1, L T}^{\varangle}\right)\right] \\
& -\sin ^{2} \theta \sin \left(\phi_{h}+2 \phi_{R}-\phi_{S}\right) \mathcal{I}\left[\frac{2\left(\vec{p}_{T} \cdot \vec{k}_{T}\right)\left(\vec{p}_{T} \cdot \hat{P}_{h \perp}\right)-\left(\vec{k}_{T} \cdot \hat{P}_{h \perp}\right) \vec{p}_{T}^{2}}{2 M^{2} M_{h}} h_{1 T}^{\perp}\left(-\frac{|\vec{R}|}{\left|\vec{k}_{T}\right|} H_{1, T T}^{\varangle}\right)\right] \\
& +\sin \theta \sin \left(4 \phi_{h}-\phi_{R}-\phi_{S}\right) \mathcal{I}\left[\left(\frac{\vec{k}_{T}^{2}\left[2\left(\vec{p}_{T} \cdot \hat{P}_{h \perp}\right)^{2}-\vec{p}_{T}^{2}\right]}{4 M^{2} M_{h}^{2}}-2\left(\vec{k}_{T} \cdot \hat{P}_{h \perp}\right)\right.\right. \\
& \left.\left.\times \frac{\left[4\left(\vec{p}_{T} \cdot \hat{P}_{h \perp}\right)^{2}\left(\vec{k}_{T} \cdot \hat{P}_{h \perp}\right)-2\left(\vec{p}_{T} \cdot \hat{P}_{h \perp}\right)\left(\vec{p}_{T} \cdot \vec{k}_{T}\right)-\vec{p}_{T}^{2}\left(\vec{k}_{T} \cdot \hat{P}_{h \perp}\right)\right]}{4 M^{2} M_{h}^{2}}\right) h_{1 T}^{\perp}\left(-\frac{2 M_{h}}{\left|\vec{k}_{T}\right|} H_{1, O T}^{\perp}\right)\right] \\
& +\sin 2 \theta \sin \left(4 \phi_{h}-\phi_{R}-\phi_{S}\right) \mathcal{I}\left[\left(\frac{\vec{k}_{T}^{2}\left[2\left(\vec{p}_{T} \cdot \hat{P}_{h \perp}\right)^{2}-\vec{p}_{T}^{2}\right]}{4 M^{2} M_{h}^{2}}-2\left(\vec{k}_{T} \cdot \hat{P}_{h \perp}\right)\right.\right. \\
& \left.\left.\times \frac{\left[4\left(\vec{p}_{T} \cdot \hat{P}_{h \perp}\right)^{2}\left(\vec{k}_{T} \cdot \hat{P}_{h \perp}\right)-2\left(\vec{p}_{T} \cdot \hat{P}_{h \perp}\right)\left(\vec{p}_{T} \cdot \vec{k}_{T}\right)-\vec{p}_{T}^{2}\left(\vec{k}_{T} \cdot \hat{P}_{h \perp}\right)\right]}{4 M^{2} M_{h}^{2}}\right) h_{1 T}^{\perp}\left(-\frac{M_{h}}{\left|\vec{k}_{T}\right|} H_{1, L T}^{\perp}\right)\right] \\
& +\sin ^{2} \theta \sin \left(5 \phi_{h}-2 \phi_{R}-\phi_{S}\right) \mathcal{I}\left[\left(\frac{2 \vec{k}_{T}^{2}\left(\vec{k}_{T} \cdot \hat{P}_{h \perp}\right)\left[2\left(\vec{p}_{T} \cdot \hat{P}_{h \perp}\right)^{2}-\vec{p}_{T}^{2}\right]}{4 M^{2} M_{h}^{3}}+\left[\vec{k}_{T}^{2}-4\left(\vec{k}_{T} \cdot \hat{P}_{h \perp}\right)^{2}\right]\right.\right. \\
& \left.\left.\left.\times \frac{\left[4\left(\vec{p}_{T} \cdot \hat{P}_{h \perp}\right)^{2}\left(\vec{k}_{T} \cdot \hat{P}_{h \perp}\right)-2\left(\vec{p}_{T} \cdot \hat{P}_{h \perp}\right)\left(\vec{p}_{T} \cdot \vec{k}_{T}\right)-\vec{p}_{T}^{2}\left(\vec{k}_{T} \cdot \hat{P}_{h \perp}\right)\right]}{4 M^{2} M_{h}^{3}}\right) h_{1 T}^{\perp}\left(-\frac{2 M_{h}^{2}}{\left|\vec{k}_{T}\right|^{2}} H_{1, T T}^{\perp}\right)\right]\right\},
\end{aligned}
$$


f. Polarized lepton beam and transversely polarized target

$$
\begin{aligned}
& d^{8} \sigma_{L T}=\sum_{a} \frac{\alpha^{2} e_{a}^{2}}{2 \pi s x y^{2}} \lambda_{e}\left|\vec{S}_{T}\right| C(y)\left\{\cos \left(\phi_{h}-\phi_{S}\right) \mathcal{I}\left[\frac{\vec{p}_{T} \cdot \hat{P}_{h \perp}}{M} g_{1 T}\left(\frac{1}{4} D_{1, O O}^{s}+\frac{3}{4} D_{1, O O}^{p}\right)\right]\right. \\
& ++\frac{1}{3}\left(3 \cos ^{2} \theta-1\right) \cos \left(\phi_{h}-\phi_{S}\right) \mathcal{I}\left[\frac{\vec{p}_{T} \cdot \hat{P}_{h \perp}}{M} g_{1 T}\left(\frac{3}{4} D_{1, L L}\right)\right] \\
& -\sin 2 \theta \cos \left(\phi_{R}-\phi_{S}\right) \mathcal{I}\left[\frac{\left(\vec{p}_{T} \cdot \vec{k}_{T}\right)}{2 M M_{h}} g_{1 T}\left(-\frac{M_{h}}{2\left|\vec{k}_{T}\right|} D_{1, L T}\right)\right] \\
& -\sin 2 \theta \cos \left(2 \phi_{h}-\phi_{R}-\phi_{S}\right) \mathcal{I}\left[\frac{2\left(\vec{p}_{T} \cdot \hat{P}_{h \perp}\right)\left(\vec{k}_{T} \cdot \hat{P}_{h \perp}\right)-\vec{p}_{T} \cdot \vec{k}_{T}}{2 M M_{h}} g_{1 T}\left(-\frac{M_{h}}{2\left|\vec{k}_{T}\right|} D_{1, L T}\right)\right] \\
& -\sin ^{2} \theta \cos \left(\phi_{h}-2 \phi_{R}+\phi_{S}\right) \mathcal{I}\left[\frac{2\left(\vec{k}_{T} \cdot \hat{P}_{h \perp}\right)\left(\vec{p}_{T} \cdot \vec{k}_{T}\right)-\vec{k}_{T}^{2}\left(\vec{p}_{T} \cdot \hat{P}_{h \perp}\right)}{2 M M_{h}^{2}} g_{1 T}\left(-\frac{M_{h}^{2}}{\left|\vec{k}_{T}\right|^{2}} D_{1, T T}\right)\right] \\
& -\sin ^{2} \theta \cos \left(3 \phi_{h}-2 \phi_{R}-\phi_{S}\right) \mathcal{I}\left[\left(\frac{4\left(\vec{k}_{T} \cdot \hat{P}_{h \perp}\right)^{2}\left(\vec{p}_{T} \cdot \hat{P}_{h \perp}\right)-2\left(\vec{k}_{T} \cdot \hat{P}_{h \perp}\right)\left(\vec{p}_{T} \cdot \vec{k}_{T}\right)}{2 M M_{h}^{2}}\right.\right. \\
& \left.\left.-\frac{\vec{k}_{T}^{2}\left(\vec{p}_{T} \cdot \hat{P}_{h \perp}\right)}{2 M M_{h}^{2}}\right) g_{1 T}\left(-\frac{M_{h}^{2}}{\left|\vec{k}_{T}\right|^{2}} D_{1, T T}\right)\right]-\sin 2 \theta \cos \left(\phi_{R}-\phi_{S}\right) \mathcal{I}\left[\frac{\left(\vec{p}_{T} \cdot \vec{k}_{T}\right)}{2 M M_{h}} f_{1 T}^{\perp}\left(\frac{|\vec{R}|}{2 M_{h}} G_{1, L T}^{\perp}\right)\right] \\
& +\sin 2 \theta \cos \left(2 \phi_{h}-\phi_{R}-\phi_{S}\right) \mathcal{I}\left[\frac{2\left(\vec{p}_{T} \cdot \hat{P}_{h \perp}\right)\left(\vec{k}_{T} \cdot \hat{P}_{h \perp}\right)-\vec{p}_{T} \cdot \vec{k}_{T}}{2 M M_{h}} f_{1 T}^{\perp}\left(\frac{|\vec{R}|}{2 M_{h}} G_{1, L T}^{\perp}\right)\right] \\
& -\sin ^{2} \theta \cos \left(\phi_{h}-2 \phi_{R}+\phi_{S}\right) \mathcal{I}\left[\frac{2\left(\vec{k}_{T} \cdot \hat{P}_{h \perp}\right)\left(\vec{p}_{T} \cdot \vec{k}_{T}\right)-\vec{k}_{T}^{2}\left(\vec{p}_{T} \cdot \hat{P}_{h \perp}\right)}{2 M M_{h}^{2}} f_{1 T}^{\perp}\left(\frac{|\vec{R}|}{2\left|\vec{k}_{T}\right|} G_{1, T T}^{\perp}\right)\right] \\
& +\sin ^{2} \theta \cos \left(3 \phi_{h}-2 \phi_{R}-\phi_{S}\right) \mathcal{I}\left[\left(\frac{4\left(\vec{k}_{T} \cdot \hat{P}_{h \perp}\right)^{2}\left(\vec{p}_{T} \cdot \hat{P}_{h \perp}\right)-2\left(\vec{k}_{T} \cdot \hat{P}_{h \perp}\right)\left(\vec{p}_{T} \cdot \vec{k}_{T}\right)}{2 M M_{h}^{2}}\right.\right. \\
& \left.\left.\left.-\frac{\vec{k}_{T}^{2}\left(\vec{p}_{T} \cdot \hat{P}_{h \perp}\right)}{2 M M_{h}^{2}}\right) f_{1 T}^{\perp}\left(\frac{|\vec{R}|}{2\left|\vec{k}_{T}\right|} G_{1, T T}^{\perp}\right)\right]\right\} .
\end{aligned}
$$

The pure $p$-wave sector of the previous cross sections corresponds to the results of spin- 1 production presented in Refs. [13, 38], once we apply the following identifications

$$
\begin{aligned}
\frac{3}{4} D_{1, O O}^{p} & =D_{1}, & \frac{3}{4} D_{1, L L} & =D_{1 L L}, \\
-\frac{M_{h}}{2\left|\vec{k}_{T}\right|} D_{1, L T} & =D_{1 L T}, & -\frac{M_{h}^{2}}{\left|\vec{k}_{T}\right|^{2}} D_{1, T T} & =D_{1 T T}, \\
\frac{|\vec{R}|}{2 M_{h}} G_{1, L T}^{\perp} & =G_{1 L T}, & \frac{|\vec{R}|}{2\left|\vec{k}_{T}\right|} G_{1, T T}^{\perp} & =G_{1 T T}, \\
\frac{3}{4} H_{1, O O}^{\perp p} & =H_{1}^{\perp}, & \frac{3}{4} H_{1, L L}^{\perp} & =H_{1 L L}^{\perp}, \\
-\frac{|\vec{R}|}{2 M_{h}} H_{1, L T}^{\varangle} & =H_{1 L T}, & -\frac{M_{h}}{\left|\vec{k}_{T}\right|} H_{1, L T}^{\perp} & =H_{1 L T}^{\perp}, \\
-\frac{|\vec{R}|}{\left|\overrightarrow{k_{T}}\right|} H_{1, T T}^{\varangle} & =H_{1 T T}, & -\frac{2 M_{h}^{2}}{\left|\vec{k}_{T}\right|^{2}} H_{1, T T}^{\perp} & =H_{1 T T}^{\perp} .
\end{aligned}
$$

Note, however, that while the functions on the left hand side contain a dependence on $z$ as well as on the invariant mass $M_{h}^{2}$, the functions on the right hand side depend only on $z$ : it is required to assume that the spin- 1 functions 
behave as resonances (Breit-Wigner shapes) in the invariant mass.

[1] J. C. Collins and G. A. Ladinsky (1994), hep-ph/9411444.

[2] R. L. Jaffe, X. Jin, and J. Tang, Phys. Rev. Lett. 80, 1166 (1998).

[3] A. Bianconi, S. Boffi, R. Jakob, and M. Radici, Phys. Rev. D62, 034008 (2000).

[4] V. Barone, A. Drago, and P. G. Ratcliffe, Phys. Rept. 359, 1 (2002).

[5] J. C. Collins, Nucl. Phys. B396, 161 (1993).

[6] M. Radici, R. Jakob, and A. Bianconi, Phys. Rev. D65, 074031 (2002).

[7] D. Boer, Nucl. Phys. B603, 195 (2001).

[8] A. Bianconi, S. Boffi, R. Jakob, and M. Radici, Phys. Rev. D62, 034009 (2000).

[9] A. Bacchetta, R. Kundu, A. Metz, and P. J. Mulders, Phys. Lett. B506, 155 (2001).

[10] A. V. Efremov and O. V. Teryaev, Sov. J. Nucl. Phys. 36, 140 (1982).

[11] X. Ji, Phys. Rev. D49, 114 (1994).

[12] M. Anselmino, M. Boglione, J. Hansson, and F. Murgia, Phys. Rev. D54, 828 (1996).

[13] A. Bacchetta and P. J. Mulders, Phys. Rev. D62, 114004 (2000).

[14] X. Artru and J. C. Collins, Z. Phys. C69, 277 (1996).

[15] D. Boer, R. Jakob, and M. Radici, In preparation.

[16] G. Abbiendi et al. (OPAL), Eur. Phys. J. C16, 61 (2000).

[17] P. Abreu et al. (DELPHI), Z. Phys. C65, 587 (1995).

[18] P. Abreu et al. (DELPHI), Phys. Lett. B406, 271 (1997).

[19] K. Ackerstaff et al. (OPAL), Z. Phys. C74, 437 (1997).

[20] K. Ackerstaff et al. (OPAL), Phys. Lett. B412, 210 (1997).

[21] R. L. Jaffe, Phys. Rev. D54, 6581 (1996).

[22] P. J. Mulders and R. D. Tangerman, Nucl. Phys. B461, 197 (1996), erratum-ibid. B484 (1996) 538..

[23] J. P. Ralston and D. E. Soper, Nucl. Phys. B152, 109 (1979).

[24] A. Bacchetta, M. Boglione, A. Henneman, and P. J. Mulders, Phys. Rev. Lett. 85, 712 (2000).

[25] J. Soffer, Phys. Rev. Lett. 74, 1292 (1995).

[26] S. J. Brodsky, D. S. Hwang, and I. Schmidt, Phys. Lett. B530, 99 (2002).

[27] A. V. Belitsky, X. Ji, and F. Yuan (2002), hep-ph/0208038.

[28] D. Boer (2001), proceedings of the International Workshop on Deep-Inelastic Scattering (DIS2001), Bologna, Italy, 27 Apr - 1 May 2001, eds. G. Bruni, G. Iacobucci and R. Nania (World Scientific, Singapore) in press, hep-ph/0106206.

[29] M. Stratmann and W. Vogelsang, Phys. Rev. D65, 057502 (2002).

[30] D. Boer, Nucl. Phys. Proc. Suppl. 105, 76 (2002).

[31] U. P. Sukhatme and K. E. Lassila, Phys. Rev. D22, 1184 (1980).

[32] J. J. Aubert et al. (EMC), Phys. Lett. B133, 370 (1983).

[33] R. L. Jaffe, X. Jin, and J. Tang, Phys. Rev. D57, 5920 (1998).

[34] A. Bacchetta and P. J. Mulders, Phys. Lett. B518, 85 (2001).

[35] J. Breitweg et al. (ZEUS), Eur. Phys. J. C12, 393 (2000).

[36] K. Ackerstaff et al. (HERMES), Eur. Phys. J. C18, 303 (2000).

[37] C. Adloff et al. (H1), Eur. Phys. J. C13, 371 (2000).

[38] A. Bacchetta, Ph.D. thesis, Vrije Universiteit Amsterdam (2002), hep-ph/0212025.

[39] J. C. Collins, Phys. Lett. B536, 43 (2002). 